\title{
VTOL UAV Transition Maneuver Using Incremental Nonlinear Dynamic Inversion
}

\author{
Zhenchang Liu $(\mathbb{D}$, Jie Guo $\mathbb{D}$, Mengting Li $\mathbb{D}$, Shengjing Tang $\mathbb{D}$, and Xiao Wang $(\mathbb{C}$ \\ School of Aerospace Engineering, Beijing Institute of Technology, Beijing 100081, China \\ Correspondence should be addressed to Jie Guo; guojie1981@bit.edu.cn
}

Received 25 April 2018; Revised 12 September 2018; Accepted 24 September 2018; Published 27 November 2018

Academic Editor: Paul Williams

Copyright ( 2018 Zhenchang Liu et al. This is an open access article distributed under the Creative Commons Attribution License, which permits unrestricted use, distribution, and reproduction in any medium, provided the original work is properly cited.

\begin{abstract}
The paper seeks to study the control system design of a novel unmanned aerial vehicle (UAV). The UAV is capable of vertical takeoff and landing (VTOL), transition flight and cruising via the technique of direct force control. The incremental nonlinear dynamic inversion (INDI) approach is adopted for the 6-DOF nonlinear and nonaffine control of the UAV. Based on the INDI control law, a method of two-layer cascaded optimal control allocation is proposed to handle the redundant and coupled control variables. For the weight selection in optimal control allocation, a dynamic weight strategy is proposed. This strategy can adjust the weight of the objective function according to the flight states and mission requirements, thus determining the optimizing direction and ensuring the rationality of the allocation results. Simulation results indicate that the UAV can track the target trajectory accurately and exhibit continuous maneuverability in transition flight.
\end{abstract}

\section{Introduction}

UAVs have increasing applications including surveillance, communications, search and rescue operations, and other military tasks. Among different flight conditions of UAVs, the $0-1000 \mathrm{~m}$ height area in cities is one of the most significant applications, where complex terrain and significant gusts arising from atmospheric turbulence exist.

This research studies a novel fixed-wing VTOL UAV with thrust vector engines, which can be applied in urban areas. The UAV can get rid of the restrictions imposed by takeoff and landing conditions and be accurately recovered by hover function; the UAV owns a larger combat range and higher flight speed by forward flight capability. A new concept of low-speed cruising is studied in this paper. For the most current VTOL aircrafts, the transition from hover to forward flight is short and stable. However, for lowspeed cruising, the transition is prolonged as a normal flight state. The vehicle can maintain transition flight for long time cruising by adopting direct force control and has favorable maneuverability. This flight mode is appropriate for vehicles flying in low-altitude complex conditions. In the meantime, under the low speed of UAV and the inefficient aerodynamic surface control during the transition flight, the incorporated control strategy of vectoring nozzles and aerodynamic surface should be adopted to control the attitude. In view of this, the thrust vector direct force control is of critical significance for transition maneuver. However, large nonlinearities, redundancies, and coupling effects arise when this technique is adopted.

In recent years, the research on VTOL UAVs is increasingly prosperous with the advancements in automatic control and the increasing popularity of UAV platforms [1]. The problem of transition maneuvers has been studied for different types of UAVs, including a fixed-wing aircraft equipped with a thrust vector engine and lift fan [1-3], tiltrotor aircraft [4-7], tail-sitter aircraft [8, 9], ducted-fan VTOL aircraft $[10,11]$, and tilt-wing aircraft $[12,13]$. A pilot auxiliary control system was designed by Francesco and Mattei [1] for a fixed-wing tiltrotor UAV, and the control logics for different flight states were specified and synthesized through adopting INDI method. The daisy-chaining logic was employed to handle the control redundancy. The autonomous transition control of two V/STOL aircrafts was studied by Xili et al. [2]. The nonlinear trajectory control strategies in longitudinal direction have been designed 
specifically for aircrafts in different types. The maneuver of a tilt quadcopter was researched by Ryll et al. [7]. The control design was basically dependent on the exact linearization of the motion equations, and the actuation redundancy was calculated through employing pseudoinverse matrices. The previous works generally decouple the longitudinal and lateral control, design control logics for hover, transition flight, and forward flight, respectively, and regard some redundant control variables as constants to solve the transition maneuver control problem. The UAV's maneuver potential with vectored thrust cannot be fully utilized.

The INDI method is adopted in this paper to control UAV's position/attitude during transition maneuver. The INDI method, which originates from the nonlinear dynamic inversion (NDI), solves the incremental form of equations of motion and generates a control law substantially reducing the dependence on aerodynamic model and other vehicle models. INDI was firstly adopted to control UAV's attitude control by Sieberling et al. [14]. The INDI attitude control law of a quadcopter was proposed, and the momentum of the propellers was incorporated in the controller by Smeur et al. [15]. They generalized this method based on the previous work to the outer control loop (position control loop) of a quadcopter under severe gust loads $[16,17]$. Lu et al. [18] applied the INDI method to the fixed-wing aircraft trajectory controller. They compared the performance of the INDI controller with that of NDI approach and proved that the INDI method can reduce the model uncertainties effectively.

An INDI control system is designed in this paper to address the 6-DOF nonlinear control of the UAV in transition flight, and the main contributions are listed below:

(1) Given the problems of strong nonlinearities and multiaxis coupling characteristics, a unified 6-DOF nonlinear control strategy is proposed to control position/ attitude and there is no need to switch the control logic according to different flight states. The INDI method is introduced to address the model uncertainty and control coupling problem. Different from the work conducted by $\mathrm{Lu}$ et al. [18], the sideslip angle $\beta$ is not assumed to be zero, and the vectored thrust in 3 directions of the body axis is considered

(2) A two-layer cascaded optimal control allocation method is proposed to address the control redundancy based on the INDI control law. The firstlayer optimal allocation is conducted to allocate the increment of flight attitude and vectored thrust in the translational dynamics control loop. The solution of the engine thrust, vectoring nozzle deflections, and aerodynamic surface deflections are calculated in the second-layer control allocation

(3) A dynamic weight selection strategy is designed for the objective function of the two-layer cascaded optimal control allocation. In dynamic weight selection strategy, a weight generator and a weight regulator are designed, which calculate weight through an analytic hierarchy process (AHP) and adjust weight according to flight states and mission requirement, respectively. The dynamic weight selection strategy can allocate control variables according to mission requirement and ensure the optimal results to be feasible

This paper is constructed as follows: the configuration and aerodynamics characteristics of the VTOL UAV researched in this paper are described in Section 2. A 6DOF mathematical model and an INDI control system of the vehicle are given in Section 3. The two-layer cascaded optimal control allocation method is presented in Section 4. Section 5 provides the simulations results. Eventually, conclusions are drawn in Section 6.

\section{UAV Configuration and Aerodynamics}

The UAV is designed in a tandem-wing plus lift-body configuration. This aerodynamic configuration can provide more lift under the limitation imposed by wing span, and it makes UAV applicable to fly in low-altitude complex flight condition. The power system consists of a lift fan in the front part of fuselage and two thrust vector engines in each side of the rear part of fuselage, as presented in Figures 1(a) and 1(b). At the bottom of the lift fan, a control rudder is equipped with the shaft. The control rudder can be deflected left and right with 12 degrees and provides lateral vectored thrust $T_{y}$ and yaw moments $n_{T}$, as illustrated in Figures 1 (c) and 1(d). The engine's vectoring nozzle can be swung up with 15 degrees and down with 90 degrees. It provides 3-axis vectored thrust for a direct force control, as shown in Figures 1(e) and 1(f). Through the cooperation of the lift fan and thrust vector engine, the UAV can realize VTOL, transition flight, and cruise.

In the case of no wind tunnel experiment, the UAV's aerodynamic coefficients were obtained by the method of aerodynamic estimation and CFD calculation together. In aerodynamic coefficients, the static pitch moment derivative $C_{\mathrm{ma}}$ ranges from -0.04 to 0.056 . When the angle of attack is from 0 to 18 degrees, $C_{\mathrm{m} \alpha}>0$, which indicates that the $\mathrm{UAV}$ is statically instable in pitch channel. The reason of instability design is that for VTOL UAV, the alignment of the mass center should be considered with power system distribution and the location of aerodynamic center, which is in the front of the mass center, facilitates the vehicle to pitch up in transition maneuver. The static yaw moment derivative $C_{n \beta}$ ranges from -0.017 to -0.03 . The static stability in yaw channel is caused by the rear wing dihedral effect. The vehicle's main dynamic moment derivatives are $C_{\mathrm{lp}}=-0.0084$, $C_{\mathrm{mq}}=-0.01739$, and $C_{\mathrm{nr}}=-0.0011$. They are all less than zero, and it indicates that the damping moments can reduce the angular velocities and keep the vehicle dynamics stable. The mapping relationship between the actuators, aerodynamic forces, and moments are usually nonlinear. In this paper, to meet the requirement of the control allocation, polynomial approximation is used to fit the aerodynamic data curve between the actuator deflections and aerodynamic coefficients. This will alleviate the need of large lookup tables 


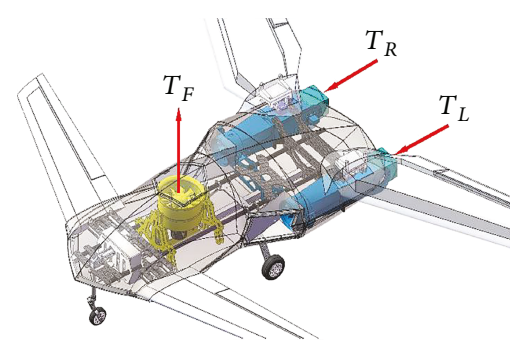

(a) Vectored thrust of power system

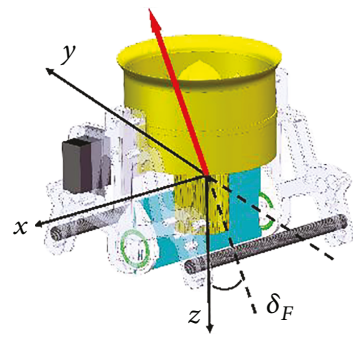

(c) Vectored thrust of lift fan

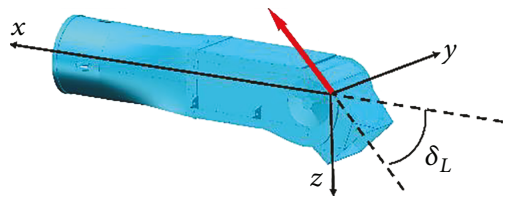

(e) Vectored thrust of engine

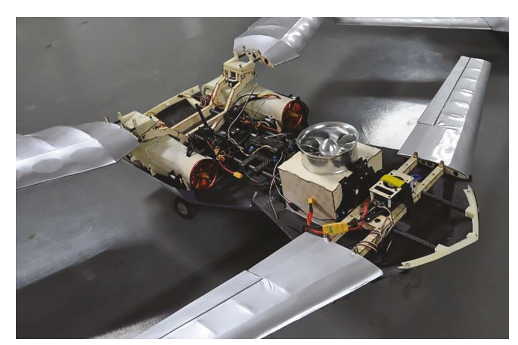

(b) UAV power system

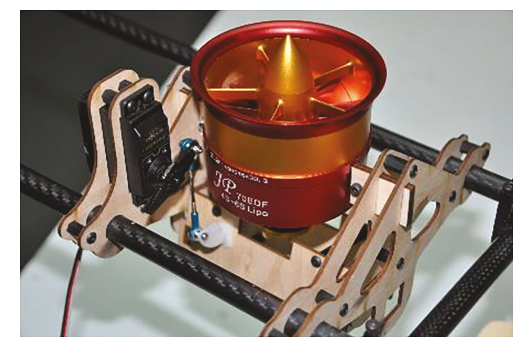

(d) Lift fan

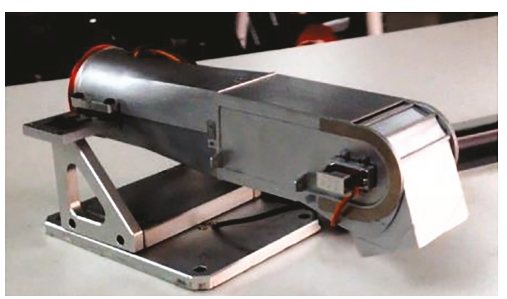

(f) Thrust vector engine

Figure 1: UAV aerodynamic and power configurations.

and speed up the control allocation computational process. The basic data of UAV is presented in Table 1.

\section{INDI Control System Design}

This section presents the design process of the control system. The trajectory tracking controller, by adopting time-scaled method, is split into four control loops, i.e., translational kinematics (position) control loop, translational dynamics (flight path) control loop, rotational kinematics (attitude) control loop, and rotational dynamics (angular rate) control loop. Given the differences of the inner and outer loops in the time constants, the control laws for inner and outer loops can be designed independently $[19,20]$. Considering that the model uncertainties caused by aerodynamic force and moments merely exist in translational dynamics and rotational dynamics control loops, these two control loops are designed by the INDI method while the other two loops are designed by the NDI method. The equations of the 6-DOF flight dynamic model and thrust vector engine model adopted to design the control system are described in this section.

3.1. Translational Kinematic Control Loop. The reference flight path vector is calculated in translational kinematic
TABle 1: Prototype basic data.

\begin{tabular}{lc}
\hline Item & Data \\
\hline Running takeoff speed & $15 \mathrm{~m} / \mathrm{s}$ \\
Cruise speed & $20 \mathrm{~m} / \mathrm{s}$ \\
Vehicle length & $1 \mathrm{~m}$ \\
Rear wing span & $1.57 \mathrm{~m}$ \\
Vehicle weight & $5 \mathrm{~kg}$ \\
Maximum engine thrust (single) & $2.6 \mathrm{~kg}$ \\
Maximum lift fan thrust & $3.8 \mathrm{~kg}$ \\
Flight range & $6 \mathrm{~km}$ \\
\hline
\end{tabular}

control loop based on the target trajectory. The position vector and flight path vector of the aircraft are defined as

$$
\begin{aligned}
& \mathbf{x}_{0}=\left[\begin{array}{lll}
x & y & z
\end{array}\right]^{\mathrm{T}}, \\
& \mathbf{x}_{1}=\left[\begin{array}{lll}
V & \chi & \gamma
\end{array}\right]^{\mathrm{T}},
\end{aligned}
$$

where $x, y$, and $z$ represent the positions of the aircraft in the North, East, and down directions, respectively. $V$ denotes the total velocity of the aircraft, $\chi$ indicates the kinematic 
azimuth angle, and $\gamma$ represents the flight path angle. The equation of translational kinematics is expressed as

$$
\dot{\mathbf{x}}_{0}=\mathbf{g}_{0}\left(\mathbf{x}_{1}\right)=\left[\begin{array}{c}
V \cos \chi \cos \gamma \\
V \sin \chi \cos \gamma \\
-V \sin \gamma
\end{array}\right] \text {. }
$$

The desired derivatives of position vector can be designed by a classical linear controller according to the reference trajectory and the feedback of the vehicle's position.

$$
\dot{\mathbf{x}}_{0}^{\mathrm{des}}=\dot{\mathbf{x}}_{0}^{\mathrm{ref}}+\mathbf{K}_{0}\left(\mathbf{x}_{0}-\mathbf{x}_{0}^{\mathrm{ref}}\right) .
$$

In (3), $\mathbf{K}_{0}=\left[\begin{array}{lll}K_{x} & K_{y} & K_{z}\end{array}\right]^{\mathrm{T}}$ are the gains of the position linear controller. For a better explanation, the definitions are given below: the variables with superscript "des" denote the desired commands generated by the linear controller, and the variables with superscript "ref" denote the reference commands given for the controller to follow. The translational kinematic control loop contains no model uncertainties, and the NDI method is adopted to calculate the control input. The reference flight path command for the next control loop is calculated in

$$
\mathbf{x}_{1}^{\mathrm{ref}}=\left[\begin{array}{c}
-\dot{Z}_{\text {des }} \\
\sin \left(\arctan \left(-\left(\dot{Z}_{\text {des }} / \dot{Y}_{\text {des }}\right) \sin \left(\arctan \left(\dot{Y}_{\text {des }} / \dot{X}_{\text {des }}\right)\right)\right)\right) \\
\arctan \frac{\dot{Y}_{\text {des }}}{\dot{X}_{\text {des }}} \\
\arctan \left(-\frac{\dot{Z}_{\text {des }}}{\dot{Y}_{\text {des }}} \sin \left(\arctan \frac{\dot{Y}_{\text {des }}}{\dot{X}_{\text {des }}}\right)\right)
\end{array}\right] .
$$

\subsection{Translational Dynamic Control Loop}

3.2.1. INDI Control Law Design. In translational dynamic control loop, the vehicle's flight path status $\mathbf{x}_{1}$ is controlled by aerodynamic force and vectored thrust together to track the reference command $\mathbf{x}_{1}^{\text {ref }}$ obtained by the last control loop. Define the control variables as

$$
\begin{aligned}
\mathbf{x}_{2} & =\left[\begin{array}{llllll}
\alpha & \beta & \mu & T_{x} & T_{y} & T_{z}
\end{array}\right]^{\mathrm{T}}, \\
\mathbf{x}_{2 a} & =\left[\begin{array}{lll}
\alpha & \beta & \mu
\end{array}\right]^{\mathrm{T}}, \\
\mathbf{x}_{2 t} & =\left[\begin{array}{lll}
T_{x} & T_{y} & T_{z}
\end{array}\right]^{\mathrm{T}} .
\end{aligned}
$$

In this loop, there are totally 6 control inputs. Among them, $\mathbf{x}_{2 a}$ represent the aerodynamic control variables, where $\mu$ is the bank angle, and $\alpha$ and $\beta$ are the angle of attack and angle of sideslip, respectively. $\mathbf{x}_{2 t}$ represent the vectored thrusts, and $T_{x}, T_{y}$, and $T_{z}$ are the components of the thrust in the body axis. The equation of translational dynamics is expressed as

$$
\begin{aligned}
\dot{\mathbf{x}}_{1} & =\mathbf{f}_{1}\left(\mathbf{x}_{1}\right)+\mathbf{g}_{1}\left(\mathbf{x}_{1}, \mathbf{x}_{2}\right), \\
\mathbf{f}_{1}\left(\mathbf{x}_{1}\right) & =\left[\begin{array}{ccc}
\frac{1}{m} & 0 & 0 \\
0 & \frac{1}{m V \cos \gamma} & 0 \\
0 & 0 & \frac{-1}{m V}
\end{array}\right] \mathbf{L}_{k g}\left[\begin{array}{c}
0 \\
0 \\
m g
\end{array}\right], \\
\mathbf{g}_{1}\left(\mathbf{x}_{1}, \mathbf{x}_{2}\right) & =\left[\begin{array}{ccc}
\frac{1}{m} & 0 & 0 \\
0 & \frac{1}{m V \cos \gamma} & 0 \\
0 & 0 & \frac{-1}{m V}
\end{array}\right] \mathbf{L}_{k b}(\alpha, \beta, \mu)\left(\mathbf{x}_{2 t}+\mathbf{L}_{k a}(\mu) \mathbf{F}_{A}\right) .
\end{aligned}
$$

In (7) and (8), $\mathbf{L}_{k g}$ is the direction cosine matrix from earth axis to flight path axis, $\mathbf{L}_{k b}(\alpha, \beta, \mu)$ is the direction cosine matrix from body axis to flight path axis, and $\mathbf{L}_{k a}(\mu)$ is the direction cosine matrix from wind axis to flight path axis. $\mathbf{F}_{A}=\left[\begin{array}{lll}-D(\alpha) & C(\beta) & -L(\alpha)\end{array}\right]^{\mathrm{T}}$ is the aerodynamic force in which $D$ represents drag, $C$ represents aerodynamic side force, and $L$ represents lift. $\mathbf{F}_{A}$ and $\mathbf{L}_{k b}(\alpha, \beta, \mu)$ and $\mathbf{L}_{k a}$ $(\mu)$ are assumed as the functions of $\mathbf{x}_{2 a}$. The multiplication of direction cosine matrixes causes severe coupling in control variables. Furthermore, in translational dynamic control loop, the error caused by the estimation of $\mathbf{F}_{A}$ will bring uncertainties in the control system. Therefore, the INDI method is adopted to address the control problem. To rewrite (6) in incremental form, it is defined that $\mathbf{x}_{1 n}$ and $\mathbf{x}_{2 n}$ represent the values of $\mathbf{x}_{1}$ and $\mathbf{x}_{2}$ in the next time step, and their relationships are described below:

$$
\begin{aligned}
& \mathbf{x}_{1 n}=\mathbf{x}_{1}+\Delta \mathbf{x}_{1}, \\
& \mathbf{x}_{2 n}=\mathbf{x}_{2}+\Delta \mathbf{x}_{2} .
\end{aligned}
$$

Applying Taylor expansion to $\dot{\mathbf{x}}_{1 n}$ at $\left(\mathbf{x}_{1}, \mathbf{x}_{2}\right)$ and with higher-order terms neglected, the result can be expressed as

$$
\begin{aligned}
\dot{\mathbf{x}}_{1 n}= & \dot{\mathbf{x}}_{1}+\frac{\partial \mathbf{f}_{1}\left(\mathbf{x}_{1}\right)}{\partial \mathbf{x}_{1}}\left(\mathbf{x}_{1 n}-\mathbf{x}_{1}\right)+\frac{\partial \mathbf{g}_{1}\left(\mathbf{x}_{1}, \mathbf{x}_{2}\right)}{\partial \mathbf{x}_{1}}\left(\mathbf{x}_{1 n}-\mathbf{x}_{1}\right) \\
& +\frac{\partial \mathbf{g}_{1}\left(\mathbf{x}_{1}, \mathbf{x}_{2}\right)}{\partial \mathbf{x}_{2}}\left(\mathbf{x}_{2 n}-\mathbf{x}_{2}\right) .
\end{aligned}
$$

In (10), the second and third terms partial to $\mathbf{x}_{1}$ are assumed much smaller than the forth term, partial to $\mathbf{x}_{2}$. This 
commonly arises from the principle of time scale separation [20]. For simplification, it is approximated that

$$
\begin{aligned}
\frac{\partial \mathbf{g}_{1}\left(\mathbf{x}_{1}, \mathbf{x}_{2}\right)}{\partial \mathbf{x}_{2}}\left(\mathbf{x}_{2 n}-\mathbf{x}_{2}\right) & =\frac{\mathbf{g}_{1}\left(\mathbf{x}_{1}, \mathbf{x}_{2}+\Delta \mathbf{x}_{2}\right)-\mathbf{g}_{1}\left(\mathbf{x}_{1}, \mathbf{x}_{2}\right)}{\Delta \mathbf{x}_{2}}\left(\mathbf{x}_{2 n}-\mathbf{x}_{2}\right) \\
& =\mathbf{g}_{1}\left(\mathbf{x}_{1}, \mathbf{x}_{2}+\Delta \mathbf{x}_{2}\right)-\mathbf{g}_{1}\left(\mathbf{x}_{1}, \mathbf{x}_{2}\right) .
\end{aligned}
$$

Replace $\dot{\mathbf{x}}_{1 n}$ with ideal derivatives of flight path vector $\dot{\mathbf{x}}_{1}^{\text {des }}$, which is calculated by the linear controller. The incremental control equation can be denoted as

$$
\dot{\mathbf{x}}_{1}^{\text {des }}-\dot{\mathbf{x}}_{1}=\mathbf{g}_{1}\left(\mathbf{x}_{1}, \mathbf{x}_{2}+\Delta \mathbf{x}_{2}\right)-\mathbf{g}_{1}\left(\mathbf{x}_{1}, \mathbf{x}_{2}\right) .
$$

To simplify the computation process, it is assumed that

$$
\Delta x_{2 i} \Delta x_{2 j}=0
$$

where $x_{2 i}$ and $x_{2 j}$ represent the elements in vector $\mathbf{x}_{2}$. Accordingly, the equation can be rewritten into the affinein-control form

$$
\dot{\mathbf{x}}_{1}^{\mathrm{des}}-\dot{\mathbf{x}}_{1}=\left[\begin{array}{cc}
\mathbf{g}_{1 a} & 0 \\
0 & \mathbf{g}_{1 t}
\end{array}\right]\left[\begin{array}{l}
\Delta \mathbf{x}_{2 a} \\
\Delta \mathbf{x}_{2 t}
\end{array}\right],
$$

where $\mathbf{g}_{1 a}$ and $\mathbf{g}_{1 t}$ are the $3 \times 3$ matrixes, representing the aerodynamic control matrix and thrust vector control matrix. The detail information of $\mathbf{g}_{1 a}$ and $\mathbf{g}_{1 t}$ is shown in Appendix. Compared with a traditional aircraft in incremental approach, the thrust vector control matrix $\mathbf{g}_{1 t}$ is additional. The elements in $\mathbf{g}_{1 t}$ are polynomials of control variables in $\mathbf{x}_{2 a}$. Thus, the control system is transferred into a linear and time variant system, and the increment of control variables is decoupled. Then the ideal incremental virtual command $\Delta \mathbf{x}_{2}$ can be calculated by

$$
\left[\begin{array}{c}
\Delta \mathbf{x}_{2 a} \\
\Delta \mathbf{x}_{2 t}
\end{array}\right]=\left[\begin{array}{cc}
\mathbf{g}_{1 a} & 0 \\
0 & \mathbf{g}_{1 t}
\end{array}\right]^{\ddagger}\left(\dot{\mathbf{x}}_{1}^{\mathrm{des}}-\dot{\mathbf{x}}_{1}\right) .
$$

In (15), the superscript “ $¥$ " represents the dynamic weight pseudoinverse method, which will be introduced in the next section. The reference command for the next control loop is obtained by

$$
\mathbf{x}_{2}^{\mathrm{ref}}=\left[\begin{array}{l}
\mathbf{x}_{2 a} \\
\mathbf{x}_{2 t}
\end{array}\right]+\left[\begin{array}{c}
\Delta \mathbf{x}_{2 a} \\
\Delta \mathbf{x}_{2 t}
\end{array}\right] .
$$

3.2.2. Flight Path Vector Derivative Acquisition. In (12), (14), and (15), $\dot{\mathbf{x}}_{1}$ can be derived by $V, \chi$, and $\gamma$, which are measured by onboard sensors. With the $\dot{\mathbf{x}}_{1}^{\text {des }}-\dot{\mathbf{x}}_{1}$ introduced, the term $\mathbf{f}_{1}\left(\mathbf{x}_{1}\right)$ is cancelled, which is the reason why INDI is referred to a sensor-based approach. This approach transfers the dependence on the model accuracy into the dependence on the sensor accuracy. In most cases, the signals were measured by sensor contain noise, and the differentiation of noisy signal amplifies the noise. To make results accurate, a filter can be adopted to abate the noise in sensor data. In this paper, a second-order filter is employed. As stated in literatures $[16,18,21]$, the washout filter can be expressed in Laplace domain as

$$
H(s)=\frac{\omega_{n}^{2}}{s^{2}+2 \zeta \omega_{n} s+\omega_{n}^{2}} .
$$

However, the filter leads to a delay which should be compensated. In the Taylor expansion shown in (10), $\dot{\mathbf{x}}_{1}$, $\mathbf{x}_{1}$, and $\mathbf{x}_{2}$ should be from the same moment. In this regard, a second-order filter is also applied for $\mathbf{x}_{2}$ to counteract the impact caused by time delay in $\mathbf{x}_{1}$ and $\dot{\mathbf{x}}_{1}$, and (12) can be rewritten in a time-synchronized form

$$
\dot{\mathbf{x}}_{1}^{\text {des }}-\dot{\mathbf{x}}_{1 f}=\mathbf{g}_{1}\left(\mathbf{x}_{1 f}, \mathbf{x}_{2 f}+\Delta \mathbf{x}_{2}\right)-\mathbf{g}_{1}\left(\mathbf{x}_{1 f}, \mathbf{x}_{2 f}\right),
$$

where subscript " $f$ " represents the filtered variable. Alternatively, other methods can also deal with the measurement delay problem, such as predictive filtering proposed by Sieberling et al. [14]. However, the prediction requires additional modeling and cannot predict disturbances. The final reference command for $\mathbf{x}_{2}^{\text {ref }}$ can be given by

$$
\mathbf{x}_{2}^{\mathrm{ref}}=\mathbf{x}_{2 f}+\Delta \mathbf{x}_{2} .
$$

3.2.3. Uncertainty Analysis. The model uncertainty stemmed from aerodynamic force in translational dynamic control loop is analyzed in this subsection. The change of aerodynamic coefficients is assumed to be primarily caused by the angle of attack $(\alpha)$ and sideslip angle $(\beta)$ for simplification. The aerodynamic coefficient can be expressed as

$$
\left\{\begin{array}{l}
C_{D}=\bar{C}_{D 0}+C_{D \alpha} \alpha, \\
C_{C}=\bar{C}_{C 0}+C_{C \beta} \beta, \\
C_{L}=\bar{C}_{L 0}+C_{L \alpha} \alpha .
\end{array}\right.
$$

In (20), $C_{D}, C_{C}$, and $C_{L}$ represent the total drag coefficient, side force coefficient, and lift coefficient, respectively; $\bar{C}_{D 0}$ represents the sum of drag coefficients without the part contributed by $\alpha ; \bar{C}_{C 0}$ represents the sum of side force coefficients without the part contributed by $\beta ; \bar{C}_{L 0}$ represents the sum of lift coefficients without the part contributed by $\alpha$; and $C_{D \alpha}, C_{C \beta}$, and $C_{L \alpha}$ represent derivative coefficients about $\alpha$ and $\beta$. These coefficients vary with the flight condition and can be assumed as constants in the calculation of each control period. The methods to obtain these parameters involve mathematic estimation and CFD analysis, as described in Section 2. The errors between the estimate coefficients and accurate coefficients make the model uncertain and assume that the uncertainty is mainly caused by $\bar{C}_{D 0}, \bar{C}_{C 0}$, and $\bar{C}_{L 0}$. As the work by $\mathrm{Lu}$ et al. [18] indicates, the kinematic roll 
angle $(\mu)$ can be calculated directly under the assumption of $\beta=0$, which largely simplifies the control equation. Additionally, coefficients $\bar{C}_{D 0}, \bar{C}_{C 0}$, and $\bar{C}_{L 0}$ can be eliminated by adopting INDI, which evidently reduces model uncertainty. However, the UAV in this paper with direct force control owns great lateral maneuverability. Therefore, $\beta$ cannot be approximated as zero, and $\mu$ is calculated through solving the control equation. As a result, coefficients $\bar{C}_{D 0}, \bar{C}_{C 0}$, and $\bar{C}_{L 0}$ arise in the third column of $\mathbf{g}_{1 a}$ and cannot be eliminated. Nevertheless, no uncertainty exists in the thrust vector control matrix $\mathbf{g}_{1 t}$.

The application of INDI in the translational dynamics control loop has two primary advantages. First, INDI control law restrains the model uncertainty caused by aerodynamic force in control matrix $\mathbf{g}_{1 a}$ and isolates its impact on direct force incremental control. Second, INDI can decouple the control variables in $\mathbf{g}_{1}\left(\mathbf{x}_{1}, \mathbf{x}_{2}\right)$ and transfer the control equation into a linear form. Accordingly, the aerodynamic force and vectored thrust control allocation are simplified, the complex numerical solution of nonlinear coupling control is avoided, and computational load of onboard control system is reduced.

3.3. Rotational Kinematic Control Loop. The objective in this control loop is to track $\mathbf{x}_{2 a}^{\mathrm{ref}}$ given in the translational dynam- ics control loop. The angular rate vector of the vehicle is defined as

$$
\mathbf{x}_{3}=\left[\begin{array}{lll}
p & q & r
\end{array}\right]^{\mathrm{T}} .
$$

In (21), $p, q$, and $r$ represent the roll, pitch, and yaw rates in body axis, respectively. No model uncertainty exists in this control loop, and the control law based on the standard NDI approach is established below:

$$
\begin{aligned}
\dot{\mathbf{x}}_{2 a} & =\mathbf{f}_{2}\left(\mathbf{x}_{2 a}\right)+\mathbf{g}_{2}\left(\mathbf{x}_{2 a}\right) \mathbf{x}_{3}, \\
\mathbf{f}_{2}\left(\mathbf{x}_{2 a}\right) & =\frac{1}{\cos \beta}\left[\begin{array}{cc}
-\cos \gamma & -\sin \mu \cos \gamma \\
-\sin \mu \cos \beta & \cos \mu \cos \gamma \cos \beta \\
\cos \mu \sin \beta & \sin \gamma \cos \beta+\sin \mu \cos \gamma \sin \beta
\end{array}\right]\left[\begin{array}{l}
\dot{\gamma} \\
\dot{\chi}
\end{array}\right], \\
\mathbf{g}_{2}\left(\mathbf{x}_{2 a}\right) & =\frac{1}{\cos \beta}\left[\begin{array}{ccc}
-\cos \alpha \sin \beta & \cos \beta & -\sin \alpha \sin \beta \\
\sin \alpha \cos \beta & 0 & -\cos \alpha \cos \beta \\
\cos \alpha & 0 & \sin \alpha
\end{array}\right] .
\end{aligned}
$$

It is noteworthy that $\dot{\gamma}, \dot{\chi}$, and $\mu$ are not directly measured onboard in this control system. They are calculated by (23) derived from (6).

$$
\begin{aligned}
& \dot{\gamma}=\frac{(\cos \alpha \sin \beta \sin \mu+\sin \alpha \cos \mu) A_{x}-\cos \beta \sin \mu A_{y}+(\sin \alpha \sin \beta \sin \mu-\cos \alpha \cos \mu) A_{z}}{V}, \\
& \dot{\chi}=\frac{(-\cos \alpha \sin \beta \cos \mu+\sin \alpha \sin \mu) A_{x}+\cos \beta \cos \mu A_{y}+(-\sin \alpha \sin \beta \cos \mu-\cos \alpha \sin \mu) A_{z}}{V \cos \gamma},
\end{aligned}
$$

where $A_{x}, A_{y}$, and $A_{z}$ are directly measured by the accelerometers of the aircraft. The reason why $\dot{\gamma}$ and $\dot{\chi}$ are not acquired by the same method adopted in translational dynamic control loop is that the filter will delay the measurement and cause errors from accurate results. The reference angular rates can be obtained by

$$
\mathbf{x}_{3}^{\mathrm{ref}}=\mathbf{g}_{2}\left(\mathbf{x}_{2 a}\right)^{-1}\left(\dot{\mathbf{x}}_{2 a}^{\mathrm{des}}-\mathbf{f}_{2}\left(\mathbf{x}_{2 a}\right)\right)
$$

where $\dot{\mathbf{x}}_{2 a}^{\text {des }}$ is the desired command and designed by linear controller, which is similar to the one adopted to design $\dot{\mathbf{x}}_{1}^{\text {des }}$.

3.4. Rotational Dynamic Control Loop. $\mathbf{x}_{4}$ is defined as the control moments acting on the vehicle, and it can be denoted as

$$
\mathbf{x}_{4}=\mathbf{x}_{4 s}+\mathbf{x}_{4 t}=\left[\begin{array}{lll}
l_{c} & m_{c} & n_{c}
\end{array}\right]^{\mathrm{T}},
$$

where $\mathbf{x}_{4 \mathrm{~s}}$ denote the moments generated by aerodynamic control surface; $\mathbf{x}_{4 \mathrm{t}}$ indicate the moments generated by vectored thrust; and $l_{c}, m_{c}$, and $n_{c}$ represent the rolling, pitching, and yawing control moments, respectively. The dynamics of the angular rates of the vehicle can be expressed into the following affine-in-control form

$$
\begin{aligned}
\dot{\mathbf{x}}_{3} & =\mathbf{f}_{3}\left(\mathbf{x}_{3}\right)+\mathbf{J}^{-1} \mathbf{x}_{4}, \\
\mathbf{f}_{3}\left(\mathbf{x}_{3}\right) & =\mathbf{J}^{-1}\left(\mathbf{M}_{a}-\mathbf{x}_{3} \times \mathbf{J} \mathbf{x}_{3}\right),
\end{aligned}
$$

where $\mathbf{J}$ denotes the inertia matrix and $\mathbf{M}_{a}$ refers to the aerodynamic moments generated by derivatives unrelated to control surface deflections.

$$
\mathbf{M}_{a}=\bar{q} S\left[\begin{array}{c}
\bar{C}_{l 0} \\
\bar{C}_{m 0} \\
\bar{C}_{n 0}
\end{array}\right] .
$$




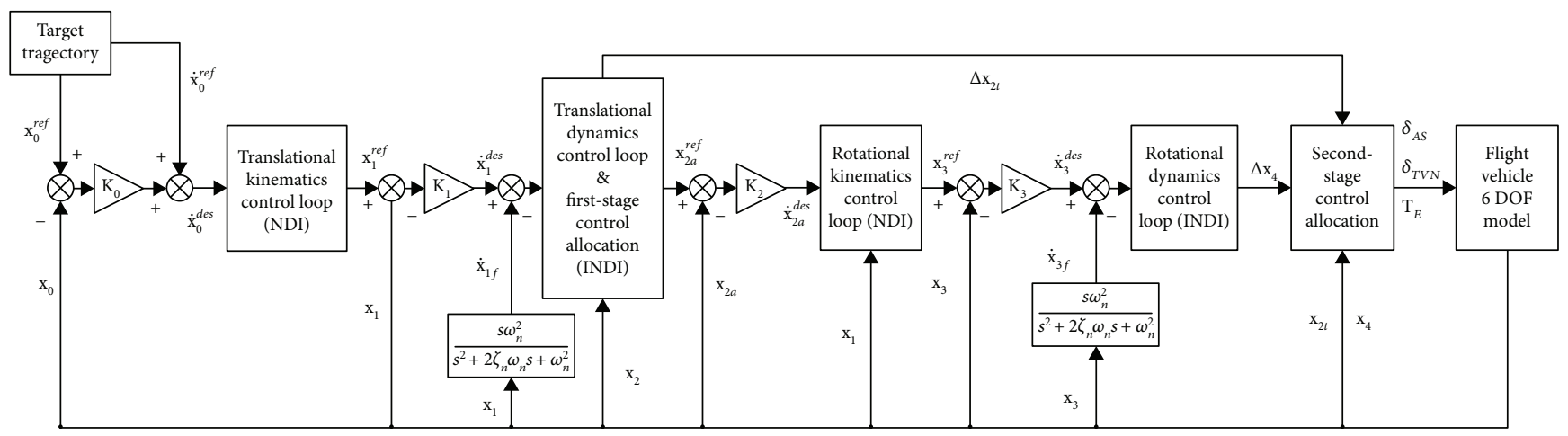

FIGURE 2: Conceptual block diagram of the control system.

Like aerodynamic force, the aerodynamic coefficients can also be denoted as

$$
\left\{\begin{array}{l}
C_{l}=\bar{C}_{l 0}+C_{l \delta_{a}} \delta_{a}+C_{l \delta_{r}} \delta_{r}, \\
C_{m}=\bar{C}_{m 0}+C_{m \delta_{e}} \delta_{e}, \\
C_{n}=\bar{C}_{n 0}+C_{n \delta_{a}} \delta_{a}+C_{n \delta_{r}} \delta_{r} .
\end{array}\right.
$$

In (27) and (28), $C_{l}, C_{m}$, and $C_{n}$ represent the total 3axis aerodynamic moment coefficients, respectively; $\bar{C}_{l 0}$ represents the sum of aerodynamic rolling moment coefficients without the part contributed by $\delta_{a}$ and $\delta_{r} ; \bar{C}_{m 0}$ represents the sum of aerodynamic pitching moment coefficients without the part contributed by $\delta_{e} ; \bar{C}_{n 0}$ represents the sum of aerodynamic yawing moment without the part contributed by $\delta_{a}$ and $\delta_{r}$; and $C_{l \delta_{a}}, C_{l \delta_{r}}, C_{m \delta_{e}}, C_{n \delta_{a}}$, and $C_{n \delta_{r}}$, represent derivative aerodynamic moment coefficients about $\delta_{a}, \delta_{e}$, and $\delta_{r}$.

The aerodynamic control moments are denoted as

$$
\begin{aligned}
\mathbf{x}_{4 s} & =\bar{q} S \mathbf{b}_{1} \mathbf{C}_{A S} \boldsymbol{\delta}_{A S}, \\
\mathbf{C}_{A S} & =\left[\begin{array}{ccc}
C_{l \delta_{a}} & 0 & C_{l \delta_{r}} \\
0 & C_{m \delta_{e}} & 0 \\
C_{n \delta_{a}} & 0 & C_{n \delta_{r}}
\end{array}\right], \\
\mathbf{b}_{1} & =\operatorname{diag}(b, \bar{c}, b),
\end{aligned}
$$

where $\bar{q}$ denotes the dynamic pressure, $S$ denotes the reference wing area, $b$ denotes the wing span, and $\bar{c}$ denotes the mean aerodynamic chord. $\boldsymbol{\delta}_{A S}=\left[\begin{array}{lll}\delta_{a} & \delta_{e} & \delta_{r}\end{array}\right]^{\mathrm{T}}$ are control surface deflections. The control moment coefficients $\mathbf{C}_{A S}$ are assumed to be accurate in aerodynamic estimation, and the uncertainties are considered to be caused principally by $\bar{C}_{l 0}, \bar{C}_{m 0}$, and $\bar{C}_{n 0}$. Rewriting (26) into the incremental form, as presented in (30), the term $\mathbf{f}_{3}\left(\mathbf{x}_{3}\right)$ can be cleared up.

$$
\Delta \mathbf{x}_{4}=\mathbf{J}\left(\dot{\mathbf{x}}_{3}^{\mathrm{des}}-\dot{\mathbf{x}}_{3 f}\right)
$$

In (30), $\dot{\mathbf{x}}_{3 f}$ represents the derivative of $\mathbf{x}_{3}$, which is the filtered signal collected by the sensor. In the absence of $\mathbf{f}_{3}\left(\mathbf{x}_{3}\right)$, the uncertainty caused by $\mathbf{M}_{a}$ is eliminated and the nonlinear cross couplings of the angular rate term is also cancelled. The reference control moments can be calculated as

$$
\mathbf{x}_{4}^{\mathrm{ref}}=\mathbf{x}_{4 f}+\Delta \mathbf{x}_{4}
$$

After the calculation of four control loops, $\Delta \mathbf{x}_{2 t}$ and $\Delta \mathbf{x}_{4}$ will be output as virtual commands to the secondlayer control allocation. The control allocation of the engine thrust, vectoring nozzles, and aerodynamic control surface deflections will be introduced in the next section. The block diagram of the control system is illustrated in Figure 2.

\section{Two-Layer Cascaded Optimal Control Allocation}

The allocation method of redundant control variables (elements in $\mathbf{x}_{2}$ and $\mathbf{x}_{4}$ ) in translational dynamics and rotational dynamics control loops is introduced in this section. Given this, the allocation result of $\mathbf{x}_{2}$ will affect $\mathbf{x}_{4}$, and the allocation results of power system actuators $T_{L}, T_{R}, T_{F} \delta_{R}, \delta_{L}$, and $\delta_{F}$ are determined by $\mathbf{x}_{2 t}$ and $\mathbf{x}_{4 t}$ synthetically. A two-layer cascade optimal control allocation method is designed to address UAV's control allocation based on INDI control law.

4.1. First-Layer Trajectory Incremental Control Allocation. The first-layer control allocation is conducted to allocate the increment of flight attitude $\Delta \mathbf{x}_{2 a}$ and vectored thrust $\Delta$ $\mathbf{x}_{2 t}$ in trajectory control.

4.1.1. Incremental Pseudoinverse Method. In translational dynamics control loop, the control equation is linearized by the INDI method. Accordingly, the dynamic weight pseudoinverse method can be used in control allocation. Based on (14), it is defined as

$$
\begin{aligned}
& \mathbf{B}=\left[\begin{array}{cc}
\mathbf{g}_{1 a} & 0 \\
0 & \mathbf{g}_{1 t}
\end{array}\right], \\
& \boldsymbol{v}=\dot{\mathbf{x}}_{1}^{\text {des }}-\dot{\mathbf{x}}_{1 f},
\end{aligned}
$$


where $\dot{\mathbf{x}}_{1 f}$ represents the derivative of $\mathbf{x}_{1}$, which is the filtered signal collected by the sensor. As the filter will delay the time in $\dot{\mathbf{x}}_{1 f}$, to keep all signals synchronized in control equations, the signal of $\mathbf{x}_{2}$ is also required to be filtered to counteract the error generated by the delay of $\dot{\mathbf{x}}_{1 f}$. Regardless of the control variable constrains, the optimal control allocation can be denoted as

$$
\begin{array}{ll}
\min _{\Delta \mathbf{x}_{2}} & \left\|\mathbf{W}_{1}\left(\Delta \mathbf{x}_{2}+\mathbf{x}_{2 f}\right)\right\|^{2}+\left\|\mathbf{W}_{2} \Delta \mathbf{x}_{2}\right\|^{2} \\
\text { s.t. } & \mathbf{B} \Delta \mathbf{x}_{2}=\mathbf{v},
\end{array}
$$

where $\Delta \mathbf{x}_{2}$ denotes the optimization variable and $\mathbf{x}_{2 f}$ and $\boldsymbol{v}$ are considered constants, they are all $3 \times 1$ vectors, and $\mathbf{W}_{1}$ and $\mathbf{W}_{2}$ indicate the diagonal matrixes of weight, which are generated by dynamic weight strategy, and will be discussed later. The first term of objective function, as shown in (33), is adopted to control the scale of control variables in $\mathbf{x}_{2}$, while the second term is employed to limit the change rates of the control variables. Equation (33) can be expanded as

$$
\begin{aligned}
\left\|\mathbf{W}_{1}\left(\Delta \mathbf{x}_{2}+\mathbf{x}_{2 f}\right)\right\|^{2}+\left\|\mathbf{W}_{2} \Delta \mathbf{x}_{2}\right\|^{2}= & \left(\Delta \mathbf{x}_{2}+\mathbf{x}_{2 f}\right)^{T} \mathbf{W}_{1}^{2}\left(\Delta \mathbf{x}_{2}+\mathbf{x}_{2 f}\right)+\mathbf{x}^{T} \mathbf{W}_{2}^{2} \Delta \mathbf{x}_{2} \\
= & \Delta \mathbf{x}_{2}^{T} \mathbf{W}_{1}^{2} \Delta \mathbf{x}_{2}+\Delta \mathbf{x}_{2}^{T} \mathbf{W}_{1}^{2} \mathbf{x}_{2 f}+\mathbf{x}_{2 f}^{T} \mathbf{W}_{1}^{2} \Delta \mathbf{x}_{2} \\
& +\mathbf{x}_{2 f}^{T} \mathbf{W}_{1}^{2} \mathbf{x}_{2 f}+\Delta \mathbf{x}_{2}^{T} \mathbf{W}_{2}^{2} \Delta \mathbf{x}_{2} .
\end{aligned}
$$

In (35), $\Delta \mathbf{x}_{2}{ }^{T} \mathbf{W}_{1}^{2} \mathbf{x}_{2 f}=\mathbf{x}_{2 f}^{T} \mathbf{W}_{1}^{2} \Delta \mathbf{x}_{2}$ and $\mathbf{x}_{2 f}^{T} \mathbf{W}_{1}^{2} \mathbf{x}_{2 f}$ are constants which can be calculated directly. Accordingly, it is deduced that (33) has the same minimizing argument as

$$
\Delta \mathbf{x}_{2}^{T} \mathbf{W}_{1}^{2} \Delta \mathbf{x}_{2}+2 \Delta \mathbf{x}_{2}^{T} \mathbf{W}_{1}^{2} \mathbf{x}_{2 f}+\Delta \mathbf{x}_{2}^{T} \mathbf{W}_{2}^{2} \Delta \mathbf{x}_{2}
$$

Define

$$
\begin{aligned}
\mathbf{W} & =\sqrt{\mathbf{W}_{1}^{2}+\mathbf{W}_{2}^{2}}, \\
\mathbf{x}_{20} & =\mathbf{W}^{-2}\left(\mathbf{W}_{1}^{2} \mathbf{x}_{2 f}\right) .
\end{aligned}
$$

Substitute (37) into (36) and incorporate with constant $\mathbf{x}_{20}^{T} \mathbf{W}^{2} \mathbf{x}_{20}$.

$$
\begin{aligned}
\Delta \mathbf{x}_{2}^{T} \mathbf{W}^{2} \Delta \mathbf{x}_{2}+2 \Delta \mathbf{x}_{2}^{T} \mathbf{W}^{2} \mathbf{x}_{20}+\mathbf{x}_{20}^{T} \mathbf{W}^{2} \mathbf{x}_{20} & =\left(\Delta \mathbf{x}_{2}+\mathbf{x}_{20}\right)^{T} \mathbf{W}^{2}\left(\Delta \mathbf{x}_{2}+\mathbf{x}_{20}\right) \\
& =\left\|\mathbf{W}\left(\Delta \mathbf{x}_{2}+\mathbf{x}_{20}\right)\right\|^{2} .
\end{aligned}
$$

Based on the foregoing deduction, the optimal problem presented in (33) is equivalence

$$
\begin{array}{ll}
\min _{\Delta \mathbf{x}_{2}} & \left\|\mathbf{W}\left(\Delta \mathbf{x}_{2}+\mathbf{x}_{20}\right)\right\|^{2} \\
\text { s.t. } & \mathbf{B} \Delta \mathbf{x}_{2}=\boldsymbol{v} .
\end{array}
$$

On that basis, the minimum norm solution of the control allocation problem is obtained as

$$
\begin{aligned}
\Delta \mathbf{x}_{2} & =\mathbf{G} \boldsymbol{v}+(\mathbf{G B}-\mathbf{I}) \mathbf{x}_{20}, \\
\mathbf{G} & =\mathbf{W}^{-1}\left(\mathbf{B} \mathbf{W}^{-1}\right)^{\dagger}
\end{aligned}
$$

In (41), superscript “ $\dagger$ ” represents the pseudoinverse operation for the matrix. In order to make the first-layer control allocation an equality constrained optimization problem, the range of $\Delta \mathbf{x}_{2}$ is not limited. Then an analytical solution is obtained, and numerical iterations are avoided, which is suitable for onboard use [22]. With the allocation results of $\Delta \mathbf{x}_{2 a}$, the $\mathbf{x}_{2 a}^{\text {ref }}$ can be calculated by (19). After adjusting $\mathbf{x}_{2 a}^{\text {ref }}$ into the right quadrant, as shown in (42), $\mathbf{x}_{2 a}^{\text {ref }}$ is transmitted into the rotational kinematics control loop.

$$
\mathbf{x}_{2 a}^{\mathrm{ref}} \in\left[\begin{array}{ll}
-0.5 \pi & 0.5 \pi
\end{array}\right]
$$

The $\Delta \mathbf{x}_{2 t}$ is considered the virtual command to transmit into the second-layer control allocation, in which $\Delta$ $\mathbf{x}_{2 t}$ and $\Delta \mathbf{x}_{4 t}$ are used to solve the values of power system actuators $T_{L}, T_{R}, T_{F} \delta_{R}, \delta_{L}$, and $\delta_{F}$.

4.1.2. Dynamic Weight Strategy. In the course of control allocation, the weight is adopted to describe the differences of control variables' significance. On the basis of the significance of control variables changing with flight states and mission requirements, a dynamic weight strategy is proposed to allocate control variables optimally and properly.

The traditional determination of control variable's weight largely depends on human experience. In this regard, there is no absolute criteria for weight determination. It is commonly difficult to judge a control variable's significance globally, while the significance between every two control variables can be easily compared. In dynamic weight strategy, the analytic hierarchy process (AHP) is adopted to synthesize comparison results of every two control variables and calculate each control variable's weight by AHP-judgment matrix. The weight matrixes in the objective function can be denoted as

$$
\begin{aligned}
& \mathbf{W}_{1}=\operatorname{diag}\left(W_{\alpha}, W_{\beta}, W_{\mu}, W_{T x}, W_{T y}, W_{T z}\right) \\
& \mathbf{W}_{2}=\operatorname{diag}\left(W_{\Delta \alpha}, W_{\Delta \beta}, W_{\Delta \mu}, W_{\Delta T x}, W_{\Delta T y}, W_{\Delta T z}\right) .
\end{aligned}
$$

In (43), each element in $\mathbf{W}_{1}$ and $\mathbf{W}_{2}$ represents the weight of the control variable corresponding to its subscript. According to different physical properties, control variables are divided into different sets. The 


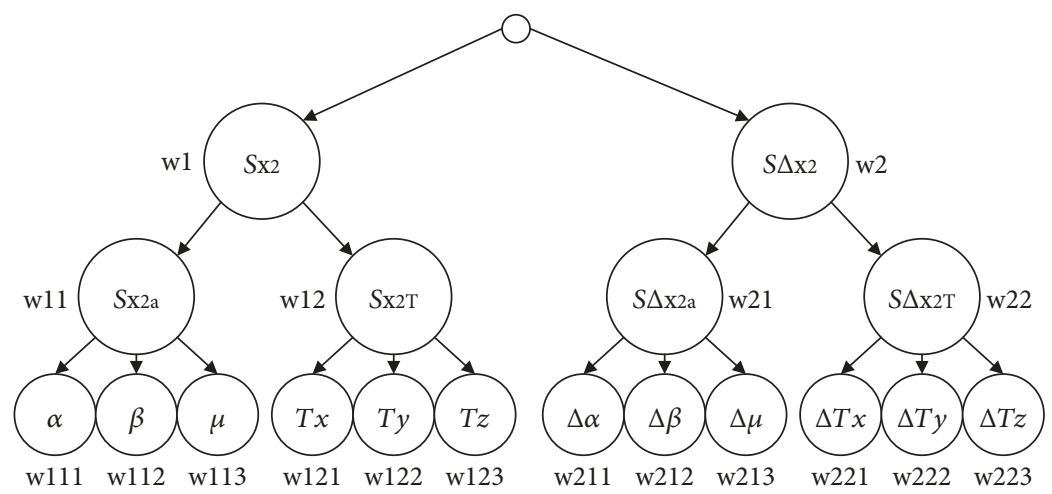

FIGURE 3: Illustration of weight structure.

definitions and subordinations, respectively, of the sets are expressed in

$$
\begin{aligned}
& \left\{\begin{array}{l}
S_{\mathbf{x}_{2}}=\left\{\alpha, \beta, \mu, T_{x}, T_{y}, T_{z}\right\}, \\
S_{\mathbf{x}_{2 a}}=\{\alpha, \beta, \mu\}, \\
S_{\mathbf{x}_{2 t}}=\left\{T_{x}, T_{y}, T_{z}\right\}, \\
S_{\Delta \mathbf{x}_{2}}=\left\{\Delta \alpha, \Delta \beta, \Delta \mu, \Delta T_{x}, \Delta T_{y}, \Delta T_{z}\right\}, \\
S_{\Delta \mathbf{x}_{2 a}}=\{\Delta \alpha, \Delta \beta, \Delta \mu\}, \\
S_{\Delta \mathbf{x}_{2 t}}=\left\{\Delta T_{x}, \Delta T_{y}, \Delta T_{z}\right\},
\end{array}\right. \\
& \left\{\begin{array}{l}
S_{\mathbf{x}_{2 a}} \subseteq S_{\mathbf{x}_{2}}, \\
S_{\mathbf{x}_{2 t}} \subseteq S_{\mathbf{x}_{\mathbf{x}}}, \\
S_{\Delta \mathbf{x}_{2 a}} \subseteq S_{\Delta \mathbf{x}_{2}}, \\
S_{\Delta \mathbf{x}_{2 t}} \subseteq S_{\Delta \mathbf{x}_{2}} .
\end{array}\right.
\end{aligned}
$$

Based on the subordinations, the sets and control variables are classified into three hierarchies, known as the weight structure, as presented in Figure 3.

The weights in different hierarchies are determined, respectively, and they all obey the rules listed below:

(1) Weights in the same set are required to be normalized

$$
\left\{\begin{array}{l}
\sum_{i=1}^{2} w_{i}=1, \\
\sum_{j=1}^{2} w_{i j}=1 \quad(i=1,2), \\
\sum_{k=1}^{3} w_{i j k}=1 \quad(i, j=1,2)
\end{array}\right.
$$

(2) Weights in the first and second hierarchies satisfy

$$
w_{1}, w_{2}, w_{11}, w_{12}, w_{21}, w_{22} \in\{0: 0.01: 1\}
$$

(3) In the third hierarchy, $\mathrm{w}_{i j k}$ is acquired by the APHjudgment matrix. The judgment matrix is established for each set, with elements representing the priorities of every two control variables in the same set. The processes of APH method are introduced in $[23,24]$. According to the weights in each hierarchy, the final weights in the objective function can be calculated by

$$
\left\{\begin{array}{l}
W_{1(k, k)}=w_{1} \times w_{11} \times w_{11 k}(k=1,2,3), \\
W_{1(k+3, k+3)}=w_{1} \times w_{12} \times w_{12 k}(k=1,2,3), \\
W_{2(k, k)}=w_{2} \times w_{21} \times w_{21 k}(k=1,2,3), \\
W_{2(k+3, k+3)}=w_{2} \times w_{22} \times w_{22 k}(k=1,2,3),
\end{array}\right.
$$

where $W_{i(k, k)}$ represents the element in weight matrix $\mathbf{W}_{i}$ row $k$ column $k$. In practical flight, the controller is required to generate the initial value of the weight structure according to the mission requirement and dynamically adjust the value of the weight structure according to the conditions of actuator saturation. In dynamic weight strategy, the weight generator and weight regulator are designed to realize the foregoing functions.

The weight generator is designed to generate the initial value of the weight structure, in every control period before optimization. Its working principle is introduced below:

Step 1. Establish weight structures which consist of $w_{i}, w_{i j}$, and $w_{i j k}$.

Step 2. Artificially design and test several weight structures according to some typical flight states and mission requirements. On this basis, index weight structures with their relative flight states and mission requirements save weight structures into repository. 
Step 3. Acquire weight structure corresponding to the current flight state and mission requirement through traversing the repository.

A simplified repository is established in this paper. For flight states, merely the impact of velocity is factored in, and velocities $5 \mathrm{~m} / \mathrm{s}, 10 \mathrm{~m} / \mathrm{s}, 15 \mathrm{~m} / \mathrm{s}, 20 \mathrm{~m} / \mathrm{s}$, and $25 \mathrm{~m} / \mathrm{s}$ count as typical flight states. In mission requirement, only the impact of direct force control level $\left(\mathrm{DFC}_{\text {level }} \in[0,1]\right)$ is factored in, and $\mathrm{DFC}_{\text {level }}$ equals $0,0.5$, and 1 count as typical mission requirements. Larger $\mathrm{DFC}_{\text {level }}$ represents less attitude changes and more direct force control, while smaller DFC level which represents less vectored thrust usage and more attitude maneuver during trajectory tracking. The repository of $3 \times 5$ weight structures employed in this paper is acquired by artificial design and computer-based simulation. In control allocation, the weight structure for the current demand is interpolated by velocity and $\mathrm{DFC}_{\text {level }}$.

The weight regulator is designed to adjust the weight when control variables exceed their limitation and ensure the rationality of allocation results. The working process of weight regulator is shown below.

Step 1. Extract control variables saturated in the last control allocation according to the feedback information. The saturated control variable can be single or multiple.

Step 2. Update saturation counter. Every control variable has a corresponding saturation counter $n_{i j k} \in N(i, j=1,2, k=$ $1,2,3)$, which is adopted to record saturation times. If the control variable is not saturated in the last allocation, its saturation counter will be established at zero.

Step 3. Adjust the weight structure in accordance with the saturation counter results. For different hierarchies of weight structure, the adjustment strategies are different, as illustrated below:

Strategy 1. For $n_{i j k} \in[1,150]$ with the interval of every 5 count times, increase the significance of the saturated control variables relative to other control variables in the identical minimum set. On that basis, update the APHjudgment matrix and recalculate $w_{i j k}$ in set. If more than one control variable gets saturated, the comparison between the saturated control variables in significance remains unchanged, and their significance is improved compared with those unsaturated.

Strategy 2. For $n_{i j k} \in[151,300]$, the weight $\left(w_{i j}\right)$ in the middle hierarchy is required to be adjusted as Strategy 1 is exercised. Take the angle of attack $\alpha$ as an example. If $\alpha$ continues to be saturated with the interval of every 5 count times, increase the weight $\left(w_{11}\right)$ of set $S_{\mathbf{x}_{2 a}}$. In (47), $w_{i j}$ is divided into 101 levels, and $\mathrm{w}_{i j}$ is improved to a new level for each weight adjustment. Assume $w_{11}=0.6$ and $w_{12}=0.4$ before the adjustment, and with the improvement of $w_{11}$, the values turn out to be $w_{11}=0.61$ and $w_{12}=0.39$.
Strategy 3. For $n_{i j k} \in[301,+\infty]$ with an interval of every 10 count times, the weight $\left(\mathrm{w}_{i}\right)$ of set $\left(S_{\mathbf{x}_{2}}\right.$ or $\left.S_{\Delta \mathbf{x}_{2}}\right)$ is required to be adjusted, as Strategy 1 and Strategy 2 are exercised. If the weight reaches the upper limit with $w_{i}, w_{i j}=1$, the weight stops increasing; if the weight reaches the lower limit with $w_{i}, w_{i j}=0$, the decrement stops.

The range of saturation counter $n_{i j k}$ and the adjustment of interval in each strategy are determined by control frequency in the weight regulator. For example, for $100 \mathrm{~Hz}$ control frequency, the control is allocated for every 0.01 second. Following the foregoing strategy, if a control variable is saturated for more than 3 seconds, the controller will adjust the weight for every hierarchy in the weight structure to get optimal control allocation to satisfy the current flight requirement. For the excessively large range of $n_{i j k}$, the weight adjustment turns out to be oversluggish. The control variables will be saturated continuously, and the vehicle will deviate from target trajectory seriously. For the excessively small range of $n_{i j k}$, the weight adjustment will be excessively sensitive. The fastchanging weight in objective function will cause vehicle oscillation and control divergence. The working process of the first-layer control allocation and dynamic weight strategy is illustrated in Figure 4.

4.2. Second-Layer Actuator Control Allocation. In the second-layer control allocation, the allocation of aerodynamic control surfaces $\left(\delta_{a}, \delta_{e}, \delta_{r}\right)$ and power system actuators $\left(T_{L}, T_{R}, T_{F}, \delta_{L}, \delta_{R}, \delta_{F}\right)$ are conducted according to the information of vectored thrust and control moments calculated in translational dynamics control loop and rotational dynamics control loops, respectively.

The dynamic model of the engine system can be denoted as

$\mathbf{x}_{2 \mathrm{t}}=\left[\begin{array}{c}T_{x} \\ T_{y} \\ T_{z}\end{array}\right]=\left[\begin{array}{c}T_{L} \cos \delta_{L}+T_{R} \cos \delta_{R} \\ T_{F} \sin \delta_{F} \\ T_{L} \sin \delta_{L}+T_{R} \sin \delta_{R}-T_{F} \cos \delta_{F}\end{array}\right]$,
$\mathbf{x}_{4 \mathrm{t}}=\left[\begin{array}{c}l_{T} \\ m_{T} \\ n_{T}\end{array}\right]=\left[\begin{array}{c}-T_{L} \sin \delta_{L} d_{y t}+T_{R} \sin \delta_{R} d_{y t} \\ \left(T_{L} \sin \delta_{L}+T_{L} \sin \delta_{L}\right) d_{x t}+T_{F} \cos \delta_{F} d_{x f} \\ \left(T_{L} \cos \delta_{L}-T_{R} \cos \delta_{R}\right) d_{y t}+T_{F} \sin \delta_{F} d_{x f}\end{array}\right]$.

Define

$$
\left\{\begin{array}{l}
\mathbf{T}_{E}=\left[\begin{array}{lll}
T_{L} & T_{R} & T_{F}
\end{array}\right]^{\mathrm{T}}, \\
\boldsymbol{\delta}_{\mathrm{TVN}}=\left[\begin{array}{lll}
\delta_{L} & \delta_{R} & \delta_{F}
\end{array}\right]^{\mathrm{T}}, \\
\boldsymbol{\delta}_{A S}=\left[\begin{array}{lll}
\delta_{a} & \delta_{e} & \delta_{r}
\end{array}\right]^{\mathrm{T}} .
\end{array}\right.
$$

In (49) and (50), $T_{F}$ denotes lift fan thrust; $T_{L}$ and $T_{R}$ denote the left and right engine thrusts, respectively; $\delta_{F}$ 


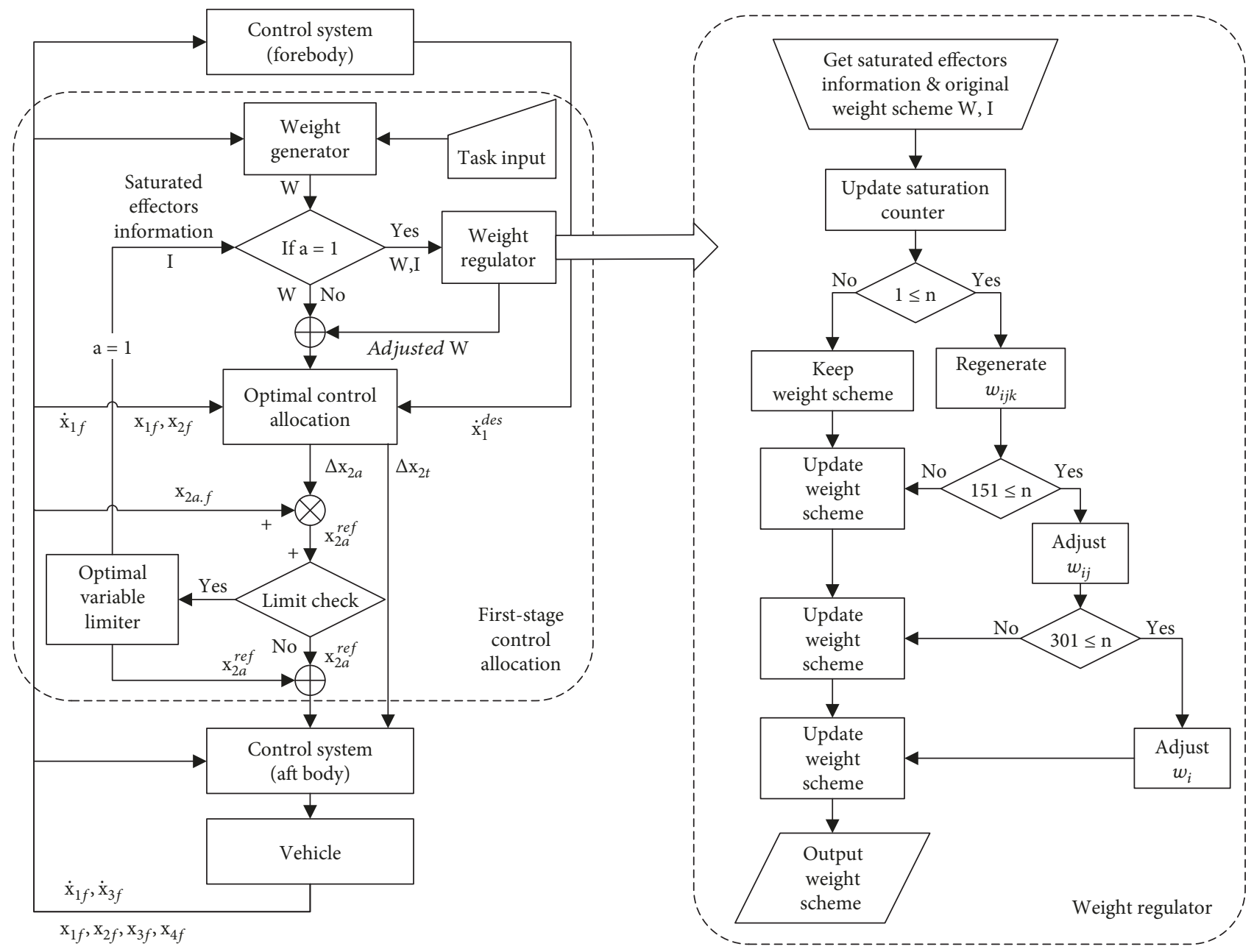

FIGURE 4: Working process of first-layer control allocation and dynamic weight strategy.

denotes the lift fan rudder deflection angle; $\delta_{L}$ and $\delta_{R}$ denote the left and right engine thrust vector nozzle deflections, respectively; $d_{x f}$ denotes the distance from the lift fan center to the vehicle mass center in body $x$ -axis; $d_{x t}$ and $d_{y t}$ denote the distance from the engine nozzle to the vehicle mass center in body $x$-axis and $y$-axis, respectively; and $\delta_{a}, \delta_{e}$, and $\delta_{r}$ represent the alerion, elevator, and rudder, respectively. To keep time synchronized, the output of the actuator model will go through a second-order filter to get $\mathbf{T}_{E . f}, \boldsymbol{\delta}_{\mathrm{TVN} . f}, \boldsymbol{\delta}_{A S . f}$, where subscript " $f$ " denotes the filtered control output. In the second-layer control allocation, the daisy chaining method is adopted to allocate aerodynamic control moment and thrust vector moment. On that basis, the control output of the power system is calculated by $\mathbf{x}_{2 t}$ and $\mathbf{x}_{4 t}$. The second-layer control allocation can be conducted in the incremental linear form or normal nonlinear form, and the two methods are introduced below.

4.2.1. Incremental Linear Allocation Method. The incremental linear allocation method allocates $\Delta \mathbf{T}_{E} \Delta \boldsymbol{\delta}_{\mathrm{TVN}}$ and $\Delta \boldsymbol{\delta}_{A S}$ according to $\Delta \mathbf{x}_{4}$ and $\Delta \mathbf{x}_{2 t}$. The incremental daisy chaining is adopted to allocate $\Delta \mathbf{x}_{4 s}$ and $\Delta \mathbf{x}_{4 t}$ based on (51). In this method, the priority of $\Delta \mathbf{x}_{4 s}$ outstrips $\Delta \mathbf{x}_{4 t}$, and thus, the thrust vector moment is adopted only when the aerodynamic control moment gets saturated.

$$
\Delta \mathbf{x}_{4}=\Delta \mathbf{x}_{4 s}+\Delta \mathbf{x}_{4 t}
$$

The increment of aerodynamic control surface is calculated by

$$
\Delta \boldsymbol{\delta}_{A S}=\frac{\Delta \mathbf{x}_{4 s}}{\bar{q} S \mathbf{b}_{1} \mathbf{C}_{A S}} .
$$

The reference value of aerodynamic control surface can be expressed as

$$
\boldsymbol{\delta}_{A S}^{r e f}=\boldsymbol{\delta}_{A S . f}+\Delta \boldsymbol{\delta}_{A S}
$$

On that basis, introduce (49) into the incremental form, as shown in (54), in which the incremental 


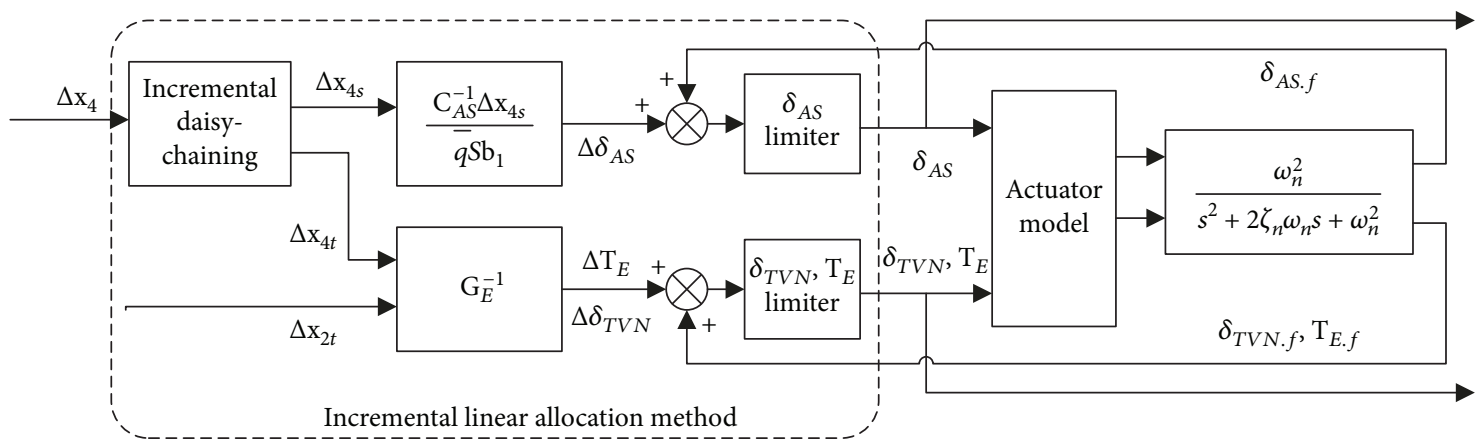

FIgURE 5: Second-layer incremental linear allocation method.

multiplication is considered a high-order small quantity and can be ignored.

$$
\begin{aligned}
& {\left[\begin{array}{c}
\Delta \mathbf{x}_{2 t} \\
\Delta \mathbf{x}_{4 t}
\end{array}\right]=\mathbf{G}_{E}\left[\begin{array}{c}
\Delta \mathbf{T}_{E} \\
\Delta \boldsymbol{\delta}_{\mathrm{TVN}}
\end{array}\right],} \\
& \mathbf{G}_{E}=\left[\begin{array}{cccccc}
\cos \delta_{L} & \cos \delta_{R} & 0 & -T_{L} \sin \delta_{L} & -T_{R} \sin \delta_{R} & 0 \\
0 & 0 & \sin \delta_{F} & 0 & 0 & T_{F} \cos \delta_{F} \\
\sin \delta_{L} & \sin \delta_{R} & -\cos \delta_{F} & T_{L} \cos \delta_{L} & T_{R} \cos \delta_{R} & T_{F} \sin \delta_{F} \\
-\sin \delta_{L} d_{y t} & \sin \delta_{R} d_{y t} & 0 & -T_{L} \cos \delta_{L} d_{y t} & T_{R} \cos \delta_{R} d_{y t} & 0 \\
\sin \delta_{L} d_{x t} & \sin \delta_{R} d_{x t} & \cos \delta_{F} d_{x f} & T_{L} \cos \delta_{L} d_{x t} & T_{R} \cos \delta_{R} d_{x t} & -T_{F} \sin \delta_{F} d_{x f} \\
\cos \delta_{L} d_{y t} & -\cos \delta_{R} d_{y t} & \sin \delta_{F} d_{x f} & -T_{L} \sin \delta_{L} d_{y t} & T_{R} \sin \delta_{R} d_{y t} & T_{F} \cos \delta_{F} d_{x f}
\end{array}\right] .
\end{aligned}
$$

The thrust vector engine model is transformed from a nonlinear system into a linear system, and $\Delta \mathbf{T}_{E}$ and $\Delta \boldsymbol{\delta}_{T V N}$ can be calculated directly by matrix inversion through the incremental linear allocation, as shown in (56). This method can evidently expedite allocation without iterative solution of nonlinear equations. However, like (14), when UAV is in high maneuver flight, the increment of control variables will be large, and the neglection of incremental multiplication will cause approximation errors. In this regard, this method is appropriate for minor or moderate maneuverable flights.

$$
\left[\begin{array}{c}
\Delta \mathbf{T}_{E} \\
\Delta \boldsymbol{\delta}_{\mathrm{TVN}}
\end{array}\right]=\boldsymbol{G}_{E}^{-1}\left[\begin{array}{c}
\Delta \mathbf{x}_{2 t} \\
\Delta \mathbf{x}_{4 t}
\end{array}\right]
$$

The reference thrust vector engine actuators are calculated by (57).

$$
\begin{gathered}
\mathbf{T}_{E}^{\mathrm{ref}}=\mathbf{T}_{E . f}+\Delta \mathbf{T}_{E}, \\
\boldsymbol{\delta}_{\mathrm{TVN}}^{\mathrm{ref}}=\boldsymbol{\delta}_{\mathrm{TVN} . f}+\Delta \boldsymbol{\delta}_{\mathrm{TVN}} .
\end{gathered}
$$

The range of $T_{L}, T_{R}, T_{F}$ and $\delta_{L}, \delta_{R}, \delta_{F}$ is limited on the basis of power system, as shown in (58). Eventually, the control signals are output to the aerodynamic control surfaces and power system.

$$
\begin{cases}T_{i}=T_{i \max }, & \text { if } T_{i} \geq T_{i \max } \\ T_{i}=T_{i \min }, & \text { if } T_{i} \leq T_{i \min } \\ \delta_{i}=\min \left(\delta_{i}\left(t_{n-1}\right)+\dot{\delta}_{i \max } \Delta t, \delta_{i} \max \right), & \text { if } \delta_{i}\left(t_{n}\right) \geq \min \left(\delta_{i}\left(t_{n-1}\right)+\dot{\delta}_{i \max } \Delta t, \delta_{i} \max \right) \\ \delta_{i}=\max \left(\delta_{i}\left(t_{n-1}\right)-\dot{\delta}_{i \max } \Delta t, \delta_{i} \min \right), & \text { if } \delta_{i}\left(t_{n}\right) \leq \max \left(\delta_{i}\left(t_{n-1}\right)-\dot{\delta}_{i \max } \Delta t, \delta_{i} \min \right)\end{cases}
$$




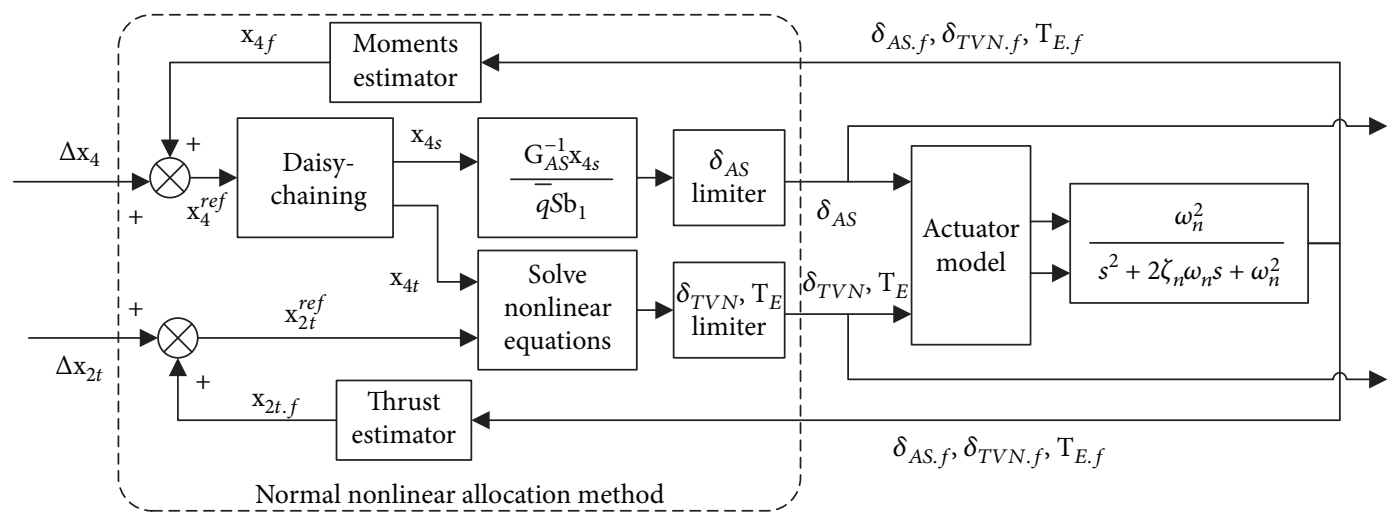

FIGURE 6: Second-layer normal nonlinear allocation method.

The flow chart of the incremental linear allocation is presented in Figure 5.

4.2.2. Normal Nonlinear Allocation Method. In nonlinear allocation, other than calculating $\Delta \mathbf{T}_{E}$ and $\Delta \boldsymbol{\delta}_{\mathrm{TVN}}$, the outputs of thrust vector engine system $\mathbf{T}_{E}$ and $\boldsymbol{\delta}_{\mathrm{TVN}}$ are calculated directly through solving nonlinear equations. This method is suitable for all maneuver flights and will not cause approximation error. The total time consumption remains rational and applicable for onboard use though the calculation time is longer than the incremental linear allocation method. The flow chart of the allocation process is exhibited in Figure 6.

\section{Simulation}

An example is designed in this section to test the control method which is discussed in the previous section. The target trajectory is designed as follows:

$$
\begin{aligned}
& v_{x}= \begin{cases}0.5 t, & 0 \leq t<20, \\
10, & 20 \leq t<60, \\
10+\frac{(t-60)}{2}, & 60 \leq t<80,\end{cases} \\
& v_{y}=\left\{\begin{array}{lc}
0, & 0 \leq t<20, \\
7 \sin \left(\frac{2 \pi(t-20)}{40}\right), & 20 \leq t<60, \\
0, & 60 \leq t<80,
\end{array}\right. \\
& v_{z}= \begin{cases}-2 \sin \left(\frac{\pi t}{20}\right), & 0 \leq t<20, \\
0, & 20 \leq t<80 .\end{cases}
\end{aligned}
$$

The initial state of UAV is static. From 0 to 20 seconds, the vehicle is climbing up and accelerating to $10 \mathrm{~m} / \mathrm{s}$ in ground $x$-axis. From 20 to 60 seconds, the UAV is in transition flight and maneuvering laterally, showing UAV's flight ability in low-altitude complex flight conditions, including cities and mountainous areas. After 60 seconds, the vehicle makes uniformly accelerated flight in ground $x$-axis and transforms from transition flight to cruise.

The example involves three different flight states, i.e., VTOL, transition flight, and cruise. These states can adequately show UAV's longitudinal and lateral maneuverability required for an urban flight vehicle. The controller designed in this paper does not need to switch as flight states change. The trajectory can be tracked merely through adjusting the weights in the controller. As the simulation results prove, the control method can solve nonlinear, nonaffine, and coupled control problems effectively and allocate redundant control variables appropriately.

To show the effectiveness of the two-layer cascaded control allocation for different flight states and mission requirements, two flight strategies are designed. In the first strategy, the UAV will track the trajectory with small attitude angle magnitude and fluctuation. In the second strategy, the vehicle is required to track the target trajectory through employing small direct force control and primarily adopting attitude control. For all strategies, the incremental linear allocation method is adopted in the secondlayer control allocation, and $0.01 \mathrm{~s}$ is adopted as the calculation step.

5.1. Strategy 1: Trajectory Tracking. For Strategy 1, the initial values of the weights are presented in (60). To avoid the errors caused by the small magnitudes of weighted control variables, the weights have been expanded by 100 times.

$$
\begin{aligned}
& \mathbf{W}_{1}=\operatorname{diag}\left[\begin{array}{llllll}
4.41 & 22.90 & 22.90 & 0.11 & 1.08 & 0.05
\end{array}\right] \\
& \mathbf{W}_{2}=\operatorname{diag}\left[\begin{array}{llllll}
9.56 & 19.13 & 19.13 & 0.24 & 0.24 & 0.24
\end{array}\right]
\end{aligned}
$$

The weight structure is generated and regulated all through the trajectory tracking, and at last, the weight changes into (61).

$$
\begin{aligned}
& \mathbf{W}_{1}=\operatorname{diag}\left[\begin{array}{llllll}
5.78 & 30.20 & 30.20 & 0.09 & 0.83 & 0.04
\end{array}\right], \\
& \mathbf{W}_{2}=\operatorname{diag}\left[\begin{array}{llllll}
6.49 & 12.95 & 12.95 & 0.19 & 0.19 & 0.19
\end{array}\right]
\end{aligned}
$$




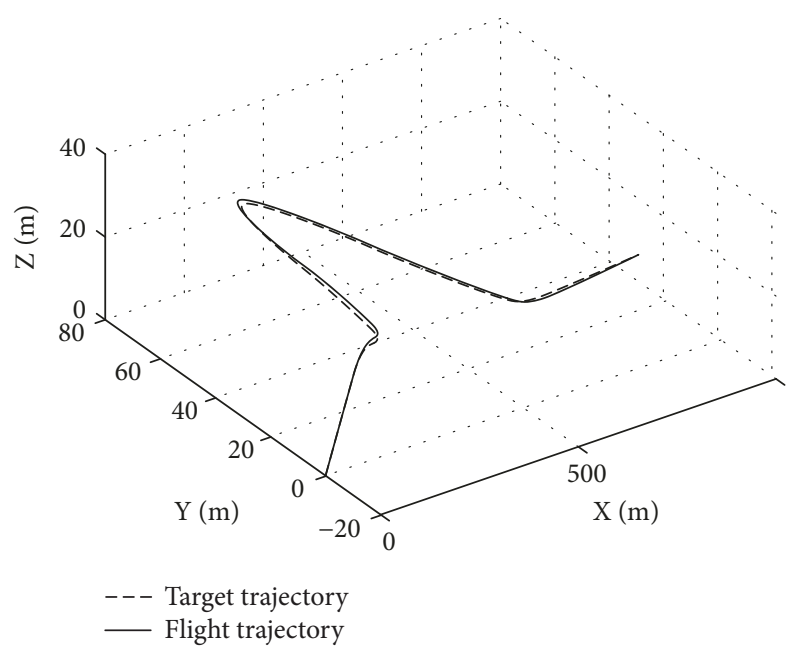

Figure 7: Trajectory tracking results.

Additionally, the weight change would be better consecutive for different flight states and mission requirements, since inconsecutive weight change will cause the saltation of flight states. The trajectory tracking result is presented in Figure 7.

The flight states of Strategy 1 are exhibited in Figure 8.

The wind angle and body angle of the UAV are presented in Figures 8(c) and 8(d). As indicated, the angle of attack $\alpha$ is negative in climbing phase, and $\alpha$ increases with the increasing of velocity and pitch angle. The UAV first keeps the minus pitch angle, and the body $z$-axis thrust is adopted to assist $x$-axis acceleration. With the rise of lift and decline of climb rate, the pitch angle $(\theta)$ and vectoring nozzle deflection angle increase, and more body $x$-axis thrust is adopted to accelerate the vehicle in ground $x$-axis. These maneuvering strategies are calculated by the controller automatically according to current flight states and weight structure.

Figures $8(\mathrm{i})$ and $8(\mathrm{j})$ show the changes of control surface deflections and aerodynamic control moments, respectively. In Figure 8(i), the black curve (delta-a) represents the aileron deflection angle $\left(\delta_{a}\right)$, and the blue curve (delta-e) represents the elevator deflection angle $\left(\delta_{e}\right)$. In Figure $8(\mathrm{j})$, the black curve (PTC-roll) represents the percentage of aerodynamic roll control moment in total roll control moments, and the blue curve (PTC-pitch) represents the percentage of aerodynamic pitch control moment in total pitch control moments. As the daisy chaining method is adopted in the second layer control allocation, the thrust vector control moments will be used only when aerodynamic control moments get saturated. Also, the effect of aerodynamic control surface is affected by the magnitude of wind angle. With large magnitude of angle of attack, the effect of control surface is less and vice versa. Therefore, in the takeoff phase, the control surfaces are in maximum deflection angle $\left( \pm 32^{\circ}\right)$. From 0 to 20 seconds, with the increase of velocity, the percentage of aerodynamic control moments increases, and control surfaces are no longer saturated.

From 20 to 60 seconds, the UAV is in transition flight and making lateral maneuvering, the flight velocity is $10 \mathrm{~m} / \mathrm{s}$, and $\alpha$ ranges from 0 degrees to 17 degrees. In Figure $8(\mathrm{f}), T_{z}$ is negative as the body $x$-axis is pointing down, indicating that UAV employs vectored thrust to compensate the lift. The change tendencies of $T_{z}$ and $\alpha$ are similar with the absolute value of velocity. As indicated, the larger lift is generated with the rise of velocity and smaller $T_{z}$ is required to compensate the lift. The change of engine thrust and nozzle deflection angle after control allocation are presented in Figures $8(\mathrm{~g})$ and $8(\mathrm{~h})$. In Figures 8(i) and $8(\mathrm{j})$, the aerodynamic control moments get saturated again when the large control moments are required, and their usage percentage decreases as the thrust vector control moments are added in. During lateral maneuver, roll angle $\phi$ (Figure $8(\mathrm{~d})$ ) and sideslip angle $\beta$ (Figure $8(\mathrm{c})$ ) are changed jointly, meaning that STT and BTT controls are adopted in the UAV simultaneously. Additionally, different values of $T_{L}$ and $T_{R}$ change similarly with $\beta$. As indicated, the UAV uses engine differential thrust to realize the STT control. The change of these flight states is corresponded to kinematic azimuth angle $\chi$, as presented in Figure 8(b). Overall, when $\chi$ is increasing, $\phi, \beta$, and $n_{c}$ keep positive; when $\chi$ is decreasing, $\phi, \beta$, and $n_{c}$ keep negative, as presented in Figures $8(\mathrm{~b}), 8(\mathrm{c}), 8(\mathrm{~d})$, and $8(\mathrm{e})$.

When $t>60 \mathrm{~s}$, with the increase of velocity, $\alpha$ and $T_{z}$ decrease, the usage percentage of aerodynamic roll control moment rises to $100 \%$, and the usage of aerodynamic pitch control moments increases. The UAV is transformed from transition flight to cruise.

5.2. Strategy 2: Trajectory Tracking. For Strategy 2, the initial values of the weights are presented in

$$
\begin{aligned}
& \mathbf{W}_{1}=\operatorname{diag}\left[\begin{array}{llllll}
2.28 & 22.78 & 22.78 & 0.13 & 1.28 & 0.12
\end{array}\right] \text {, } \\
& \mathbf{W}_{1}=\operatorname{diag}\left[\begin{array}{llllll}
9.96 & 19.92 & 19.92 & 0.28 & 0.28 & 0.28
\end{array}\right] \text {. }
\end{aligned}
$$

With the generation and regulation of weight structure during trajectory tracking, at the end of simulation, the weight changes into (63).

$$
\begin{aligned}
& \mathbf{W}_{1}=\operatorname{diag}\left[\begin{array}{llllll}
1.81 & 32.53 & 32.53 & 0.08 & 0.81 & 0.08
\end{array}\right] \\
& \mathbf{W}_{1}=\operatorname{diag}\left[\begin{array}{llllll}
6.32 & 12.65 & 12.65 & 0.18 & 0.18 & 0.18
\end{array}\right] .
\end{aligned}
$$

The flight states of Strategy 2 are presented in Figure 9.

Due to different weight selections, the tracking maneuver in Strategy 2 differs from that in Strategy 1 evidently. In Strategy 2, $\alpha$ and $\theta$ increase quickly (Figures 9(a) and 9(b)) during takeoff, and the vehicle climbs and accelerates in forward with the thrust in body $x$-axis. In the lateral maneuver process, the ranges of attitude angle and control moments (Figures 9(a) and 9(c)) in Strategy 2 are larger than the one in Strategy 1 evidently. However, the vectored thrust $T_{Z}$ and power system actuators $T_{F}, \delta_{L}$, and $\delta_{R}$ adopted in Strategy 2 (Figures 9(d), 9(e), and 9(f)) are less than those in Strategy 1 (Figures 8(f), $8(\mathrm{~g})$, and $8(\mathrm{~h}))$. Compared with Strategy 1, Strategy 2 adopts less direct force control, and the maneuver is dominated by attitude control. In the meantime, similar with Strategy 1, BTT and STT controls are both adopted in Strategy 2. In 


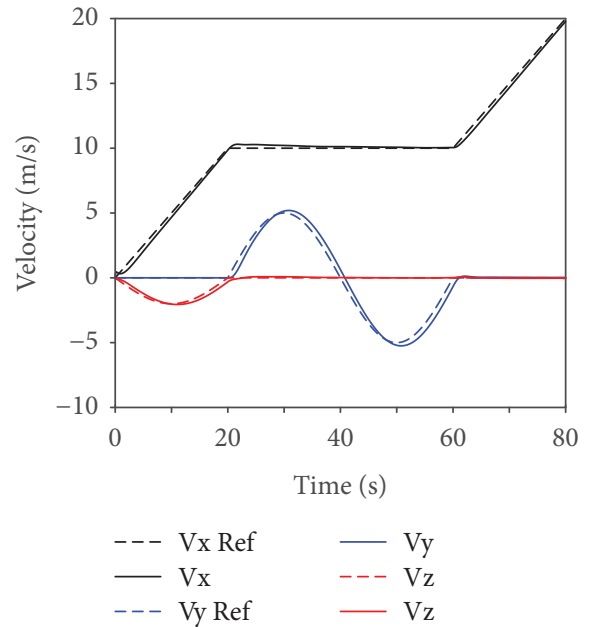

(a) Three-axis velocity

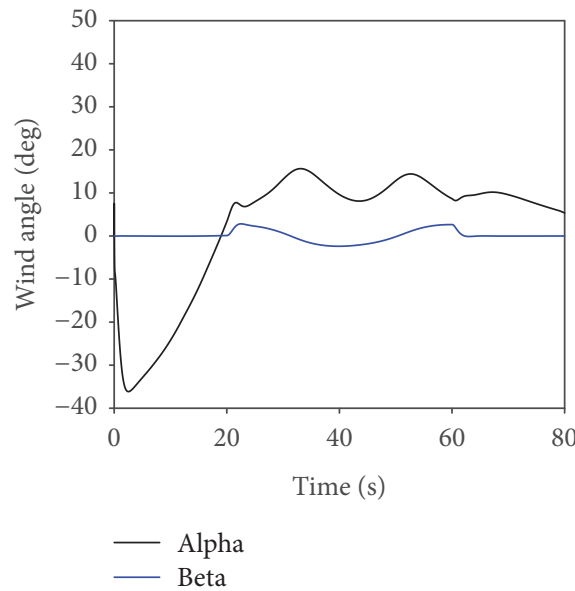

(c) Wind angle

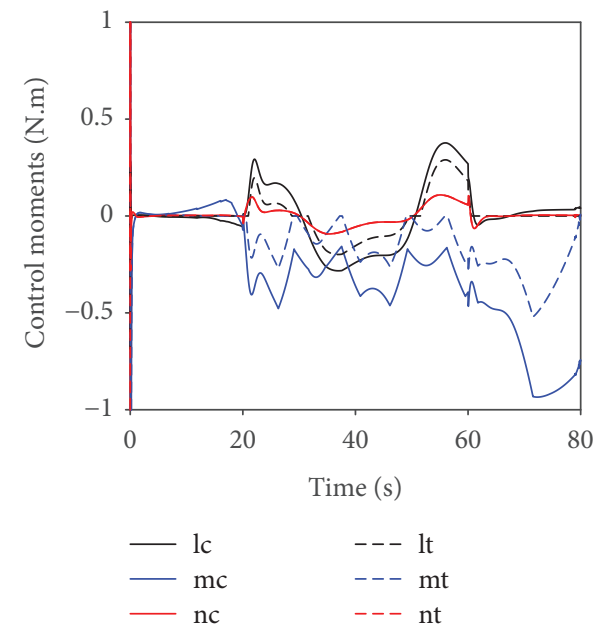

(e) Control moments

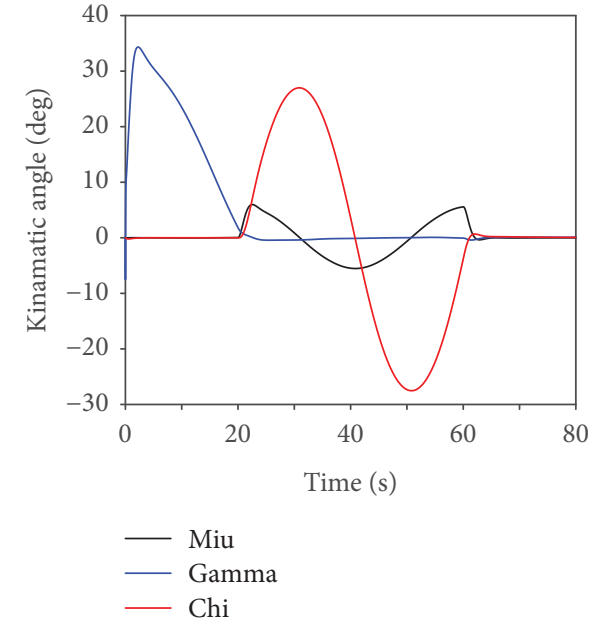

(b) Kinematic angle

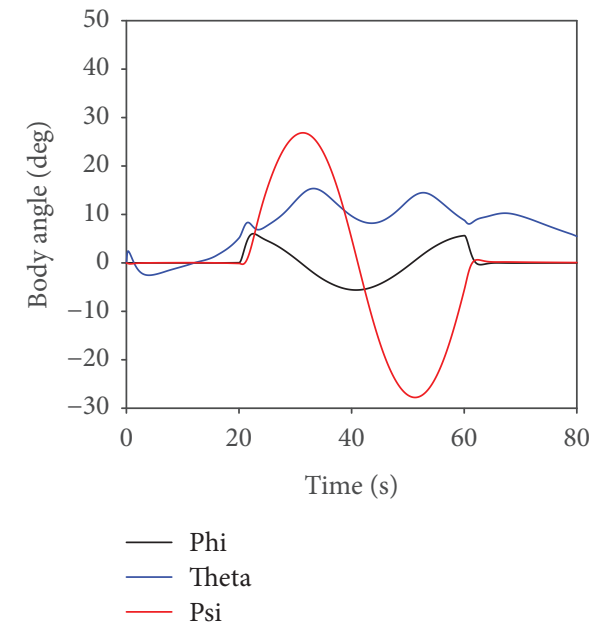

(d) Body angle

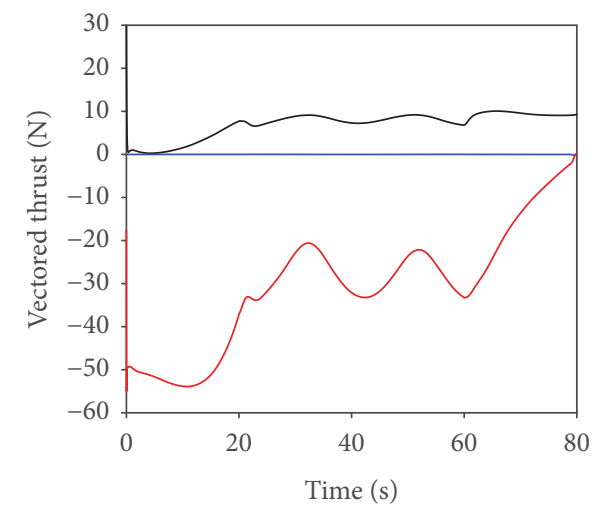

$\mathrm{Tx}$
$-\mathrm{Ty}$
$-\mathrm{Tz}$

(f) Vectored thrust in body axis

Figure 8: Continued. 


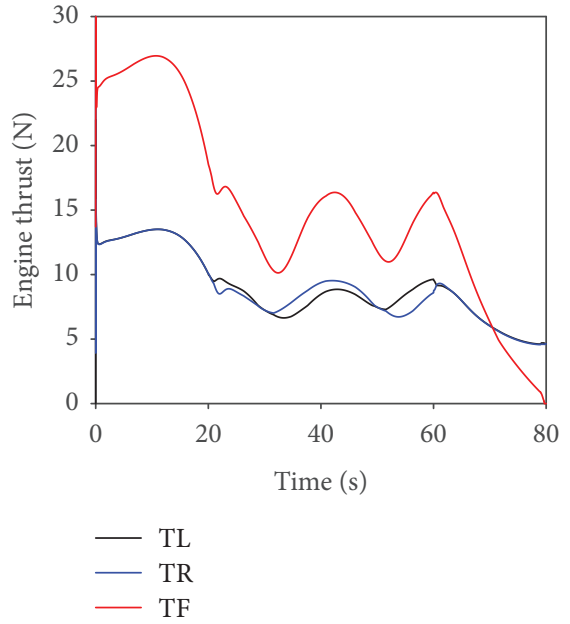

(g) Engine thrust

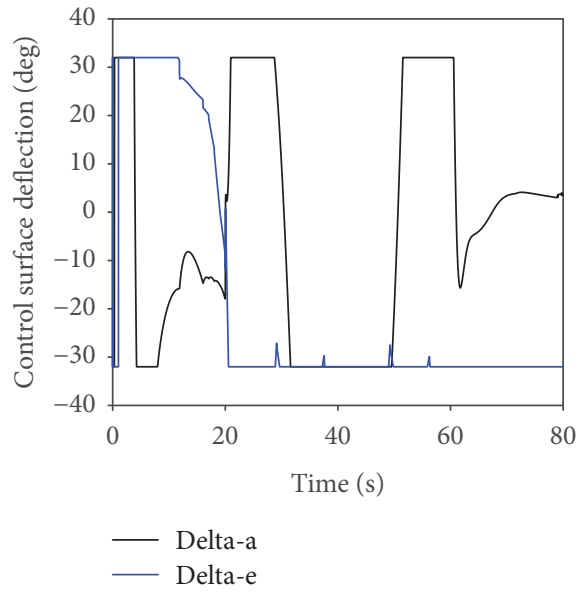

(i) Control surface deflection

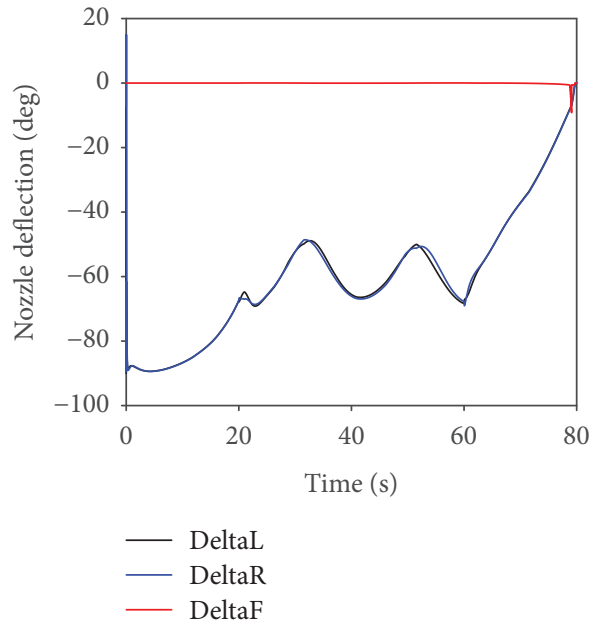

(h) Vectoring nozzle deflection

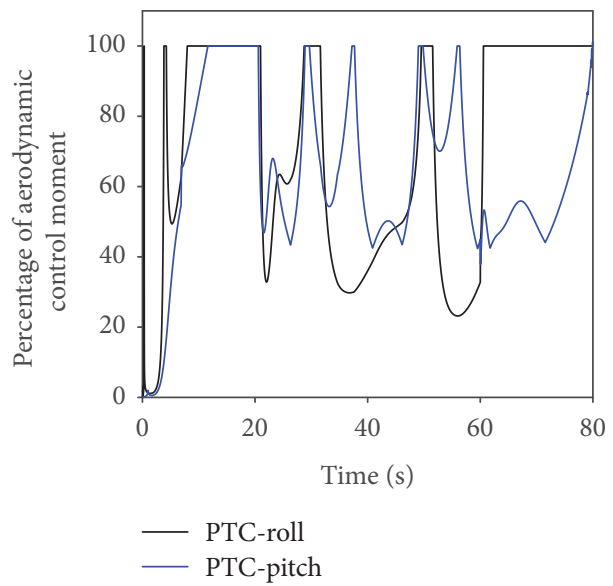

(j) Percentage of aerodynamic control moments

FIgURE 8: Flight states of Strategy 1.

Figure 9(c), from 60 seconds to 80 seconds, the saltation of control moment $m_{c}$ exists, which is caused by the aerodynamic interference between the tandem wings. During this period, $\alpha$ decreases from 36 degrees to 5 degrees. When $\alpha$ is around 18 degrees, the first saltation of pitch moment coefficient $C_{m}$ is caused by the different stall angles between the front wing and the rear wing. When $\alpha$ is around 9 degrees, the wake flow of the front wing beats on the rear wing directly, which leads to the second saltation of $C_{m}$. As indicated, in the INDI control system, the control moment $m_{c}$ is used to counteract the aerodynamic disturbances and keep the stability of flight attitude.

Comparing the change of $\alpha$ in Figures 8(c) and 9(a), we can find that when UAV makes lateral maneuver, $\alpha$ in Strategy 1 increases with the increase of $V$, while Strategy 2 is on the contrary. This is because the average $\alpha$ approaching to 12 degrees is small in Strategy 1 and does not reach the stall angle. With the rise of velocity, the increment of $\alpha$ can largely increase lift and reduce $T_{Z}$. This maneuver strategy based on weight selection is an optimal result, which makes the objective function get minor results. In Strategy 2, the average value of $\alpha$ is around
34 degrees, which exceeds the stall angle evidently. In this condition, increasing $\alpha$ will result in lift loss. Accordingly, with the increase of velocity, more lift is generated, and reducing $\alpha$ is the best solution for optimal control allocation.

The application of the INDI control method has a requirement for the frequency of the control system. High control frequency is especially required for fiercer maneuver. In INDI control, the multiplication of increment caused by coupled control variables counts as a high-order small quantity and is neglected, which will cause large errors under fierce maneuver and large sampling time. Also, in the discrete differentiator, which is used to calculate the derivative of control vectors, the larger sampling time will result in less derivation accuracy. These errors can be restrained through reducing the sampling time, which is equivalent to increase the control frequency. As the simulation results indicate, UAV can track the target trajectory accurately. The application of the INDI method and two-layer cascaded optimal control allocation in a controller of $100 \mathrm{~Hz}$ can satisfy the need of maneuver in this case. Furthermore, the simulation validates the effectiveness of the control method developed in this paper. 


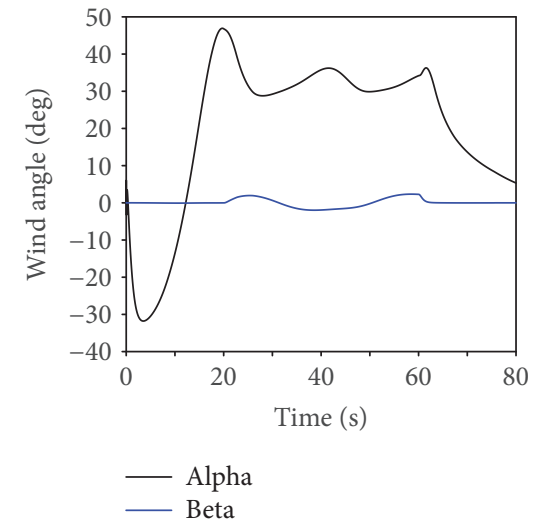

(a) Wind angle

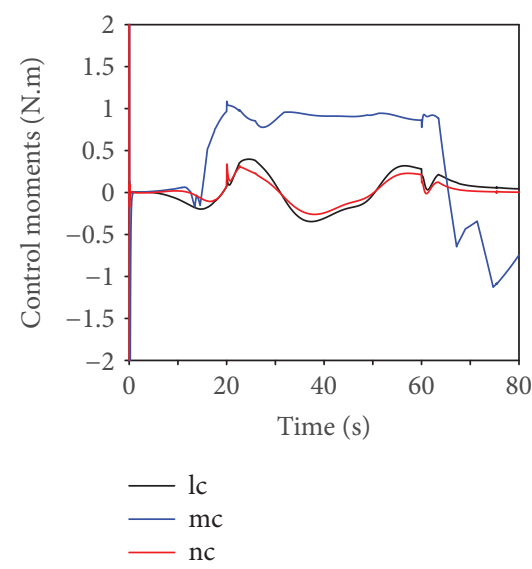

(c) Control moments

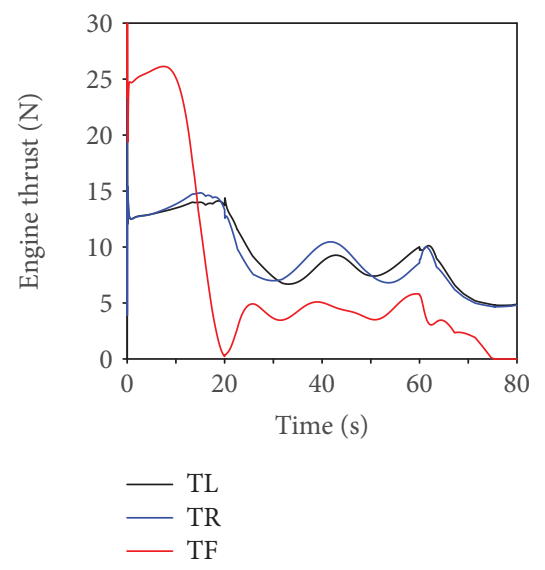

(e) Engine thrust

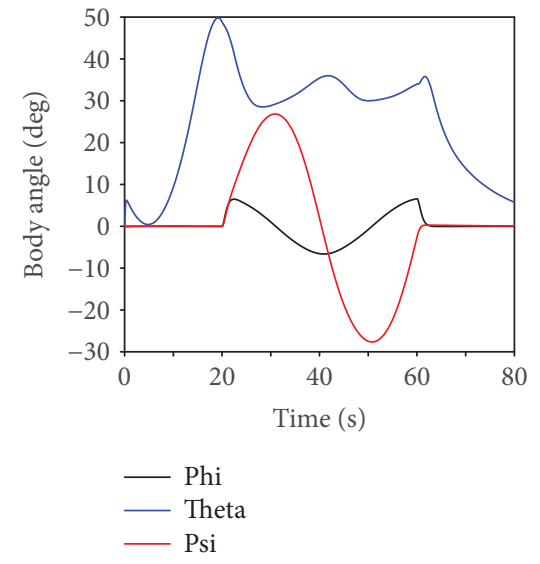

(b) Body angle

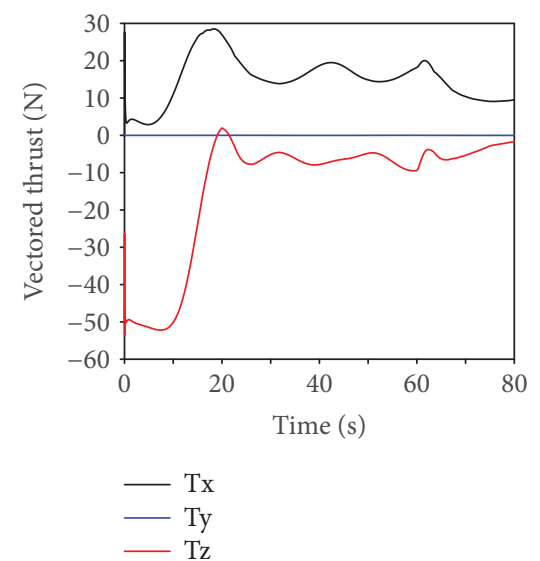

(d) Vectored thrust in body axis

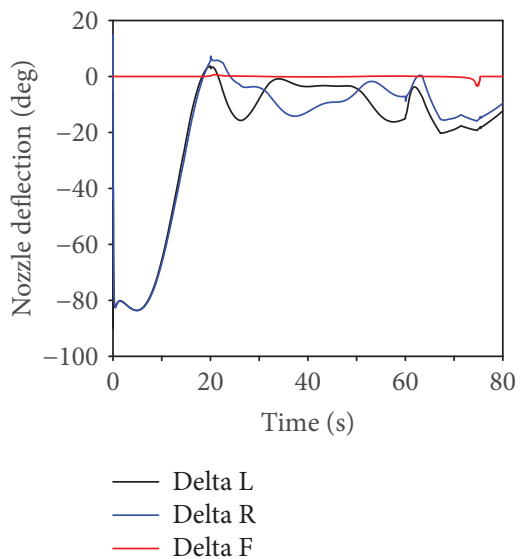

(f) Vectoring nozzle deflection

Figure 9: Flight states of Strategy 2.

\section{Conclusions}

A trajectory control system is proposed in this paper to solve the nonlinear, nonaffine, redundant, and coupled control problems existing in a UAV, which adopts direct force control technique. The research results are concluded below: (1) as the simulation indicates, the INDI controller is applicable for different flight modes, and the UAV with the INDI controller exhibits continuous maneuverability in transition flight. (2) The two-layer cascaded optimal control allocation is designed based on INDI control law; with rationally designed constraints and cascaded solving strategy, this method transforms the nonlinear coupled allocation problem into an incremental linear-equality constraint optimization problem and largely improves the allocation speed. (3) The dynamic weight strategy can adjust the weights according 
to different flight states and mission requirements, which ensures the rationality of control allocation. In the future, the control system presented in this paper will be incorporated with the controller of a prototype. A flight test is required to be further conducted to verify the effectiveness of the proposed control system.

\section{Appendix}

$$
\begin{aligned}
& \mathbf{g}_{1 a}=\left[\begin{array}{lll}
g_{1 a(11)} & g_{1 a(12)} & g_{1 a(13)} \\
g_{1 a(21)} & g_{1 a(22)} & g_{1 a(23)} \\
g_{1 a(31)} & g_{1 a(32)} & g_{1 a(33)}
\end{array}\right] \\
& g_{1 a(11)}=-T_{x} \sin \alpha \cos \beta+T_{z} \cos \alpha \cos \beta-\frac{1}{2} \rho V^{2} S C_{L \alpha} \text {, } \\
& g_{1 a(12)}=-T_{x} \sin \beta \cos \alpha+T_{y} \cos \beta-T_{z} \sin \beta \sin \alpha, \\
& g_{1 a(13)}=0 \text {, } \\
& g_{1 a(21)}=T_{x}(\sin \alpha \sin \beta \cos \mu+\cos \alpha \sin \mu)+T_{z}\left(-\cos \alpha \sin \beta \cos \mu_{*}+\sin \alpha \sin \mu\right)+\sin \mu \frac{1}{2} \rho V^{2} S C_{L \alpha} \text {, } \\
& g_{1 a(22)}=-T_{x} \cos \alpha \cos \beta \cos \mu-T_{y} \sin \beta \cos \mu-T_{z} \sin \alpha \cos \beta \cos \mu+\cos \mu \frac{1}{2} \rho V^{2} S C_{C \beta}, \\
& g_{1 a(23)}=T_{x}(\cos \alpha \sin \beta \sin \mu+\sin \alpha \cos \mu)-T_{y} \sin \mu \cos \beta+T_{z}(\sin \alpha \sin \beta \sin \mu-\cos \alpha \cos \mu) \\
& -\sin \mu \frac{1}{2} \rho V^{2} S\left(\bar{C}_{C 0}+C_{C \beta} \beta\right)+\cos \mu \frac{1}{2} \rho V^{2} S\left(\bar{C}_{L 0}+C_{L \alpha} \alpha\right), \\
& g_{1 a(31)}=T_{x}(-\cos \alpha \sin \beta \cos \mu+\sin \alpha \sin \beta \sin \mu-\cos \alpha \cos \mu)+T_{z}(-\cos \alpha \sin \beta \sin \mu-\sin \alpha \cos \mu)-\cos \mu \frac{1}{2} \rho V^{2} S C_{L \alpha} \text {, } \\
& g_{1 a(32)}=-T_{x} \cos \alpha \cos \beta \sin \mu-T_{y} \sin \beta \sin \mu-T_{z} \sin \alpha \cos \beta \sin \mu+\sin \mu \frac{1}{2} \rho V^{2} S C_{C \beta} \text {, } \\
& g_{1 a(33)}=T_{x} \sin \alpha \sin \mu+T_{y} \sin \alpha \sin \mu+T_{z}(-\sin \alpha \sin \beta \cos \mu-\cos \alpha \sin \mu) \\
& +\cos \mu \frac{1}{2} \rho V^{2} S\left(\bar{C}_{C 0}+C_{C \beta} \beta\right)+\sin \mu \frac{1}{2} \rho V^{2} S\left(\bar{C}_{L 0}+C_{L \alpha} \alpha\right), \\
& \mathbf{g}_{1 t}=\left[\begin{array}{ccc}
\cos \alpha \cos \beta & \sin \beta & \sin \alpha \cos \beta \\
-\cos \alpha \sin \beta \cos \mu+\sin \alpha \sin \mu & \cos \mu \cos \beta & -\sin \alpha \sin \beta \cos \mu-\cos \alpha \sin \mu \\
-\cos \alpha \sin \beta \sin \mu-\sin \alpha \cos \mu & \sin \mu \cos \beta & -\sin \alpha \sin \beta \sin \mu+\cos \alpha \cos \mu
\end{array}\right] \text {. }
\end{aligned}
$$

\section{Data Availability}

The results.mat data used to support the findings of this study are available from the corresponding author upon request.

\section{Conflicts of Interest}

The authors declare that there is no conflict of interest regarding the publication of this paper.

\section{Acknowledgments}

This work is supported by the Aeronautical Science Foundation of China Grant 2016ZA72001 and the National Natural Science Foundation of China Grant 11572036. The authors would like to thank all members of the Microraptor Works, Key Lab of Dynamics and Control of Flight Vehicle, Ministry of Education, School of Aerospace Engineering, Beijing Institute of Technology.

\section{References}

[1] G. Di Francesco and M. Mattei, "Modeling and incremental nonlinear dynamic inversion control of a novel unmanned tiltrotor," Journal of Aircraft, vol. 53, no. 1, pp. 73-86, 2016.

[2] Y. Xili, F. Yong, and Z. Jihong, "Transition flight control of two vertical/short takeoff and landing aircraft," Journal of Guidance, Control, and Dynamics, vol. 31, no. 2, pp. 371-385, 2008.

[3] Y. O. Aktas, U. Ozdemir, Y. Dereli et al., "Rapid prototyping of a fixed-wing VTOL UAV for design testing," Journal of Intelligent \& Robotic Systems, vol. 84, no. 1-4, pp. 639-664, 2016.

[4] M. D. Hua, T. Hamel, P. Morin, and C. Samson, "Control of VTOL vehicles with thrust-tilting augmentation," Automatica, vol. 52, pp. 1-7, 2015.

[5] P. Hartmann, C. Meyer, and D. Moormann, "Unified velocity control and flight state transition of unmanned tilt-wing aircraft," Journal of Guidance, Control, and Dynamics, vol. 40, no. 6, pp. 1348-1359, 2017. 
[6] E. Cetinsoy, C. Hancer, K. T. Oner, E. Sirimoglu, and M. Unel, "Aerodynamic design and characterization of a quad tilt-wing UAV via wind tunnel tests," Journal of Aerospace Engineering, vol. 25, no. 4, pp. 574-587, 2012.

[7] M. Ryll, H. H. Bülthoff, and P. R. Giordano, "A novel overactuated quadrotor unmanned aerial vehicle: modeling, control, and experimental validation," IEEE Transactions on Control Systems Technology, vol. 23, no. 2, pp. 540-556, 2015.

[8] D. Kubo and S. Suzuki, "Tail-sitter vertical takeoff and landing unmanned aerial vehicle: transitional flight analysis," Journal of Aircraft, vol. 45, no. 1, pp. 292-297, 2008.

[9] A. Banazadeh and N. Taymourtash, "Optimal control of an aerial tail sitter in transition flight phases," Journal of Aircraft, vol. 53, no. 4, pp. 914-921, 2016.

[10] R. Naldi and L. Marconi, "Optimal transition maneuvers for a class of V/STOL aircraft," Automatica, vol. 47, no. 5, pp. 870-879, 2011.

[11] R. Naldi and L. Marconi, "Robust control of transition maneuvers for a class of V/STOL aircraft," Automatica, vol. 49, no. 6, pp. 1693-1704, 2013.

[12] A. Maqsood and T. H. Go, "Optimization of hover-to-cruise transition maneuver using variable-incidence wing," Journal of Aircraft, vol. 47, no. 3, pp. 1060-1064, 2010.

[13] A. Maqsood and T. H. Go, "Optimization of transition maneuvers through aerodynamic vectoring," Aerospace Science and Technology, vol. 23, no. 1, pp. 363-371, 2012.

[14] S. Sieberling, Q. P. Chu, and J. A. Mulder, "Robust flight control using incremental nonlinear dynamic inversion and angular acceleration prediction," Journal of Guidance, Control, and Dynamics, vol. 33, no. 6, pp. 1732-1742, 2010.

[15] E. J. J. Smeur, G. C. H. E. D. Croon, and Q. Chu, "Gust disturbance alleviation with incremental nonlinear dynamic inversion," in 2016 IEEE/RSJ International Conference on Intelligent Robots and Systems (IROS), Daejeon, Korea, October 2016.

[16] E. J. J. Smeur, Q. Chu, and G. C. H. E. de Croon, "Adaptive incremental nonlinear dynamic inversion for attitude control of micro air vehicles," Journal of Guidance, Control, and Dynamics, vol. 39, no. 3, pp. 450-461, 2016.

[17] E. J. J. Smeur, G. C. H. E. de Croon, and Q. Chu, "Cascaded incremental nonlinear dynamic inversion for MAV disturbance rejection," Control Engineering Practice, vol. 73, pp. 79-90, 2018.

[18] P. Lu, E. J. van Kampen, C. de Visser, and Q. Chu, "Aircraft fault-tolerant trajectory control using incremental nonlinear dynamic inversion," Control Engineering Practice, vol. 57, pp. 126-141, 2016.

[19] J. Reiner, G. J. Balas, and W. L. Garrard, "Robust dynamic inversion for control of highly maneuverable aircraft," Journal of Guidance, Control, and Dynamics, vol. 18, no. 1, pp. 18-24, 1995.

[20] P. Simplício, M. D. Pavel, E. van Kampen, and Q. P. Chu, “An acceleration measurements-based approach for helicopter nonlinear flight control using incremental nonlinear dynamic inversion," Control Engineering Practice, vol. 21, no. 8, pp. 1065-1077, 2013.

[21] B. J. Bacon, A. J. Ostroff, and S. M. Joshi, "Reconfigurable NDI controller using inertial sensor failure detection \& isolation," IEEE Transactions on Aerospace and Electronic Systems, vol. 37, no. 4, pp. 1373-1383, 2001.
[22] J. Jin, "Modified pseudoinverse redistribution methods for redundant controls allocation," Journal of Guidance, Control, and Dynamics, vol. 28, no. 5, pp. 1076-1079, 2005.

[23] T. L. Saaty, "Decision making with the analytic hierarchy process," International Journal of Services Sciences, vol. 1, no. 1, pp. 83-98, 2008.

[24] O. S. Vaidya and S. Kumar, "Analytic hierarchy process: an overview of applications," European Journal of Operational Research, vol. 169, no. 1, pp. 1-29, 2006. 


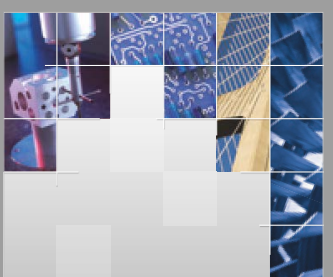

\section{Enfincering}
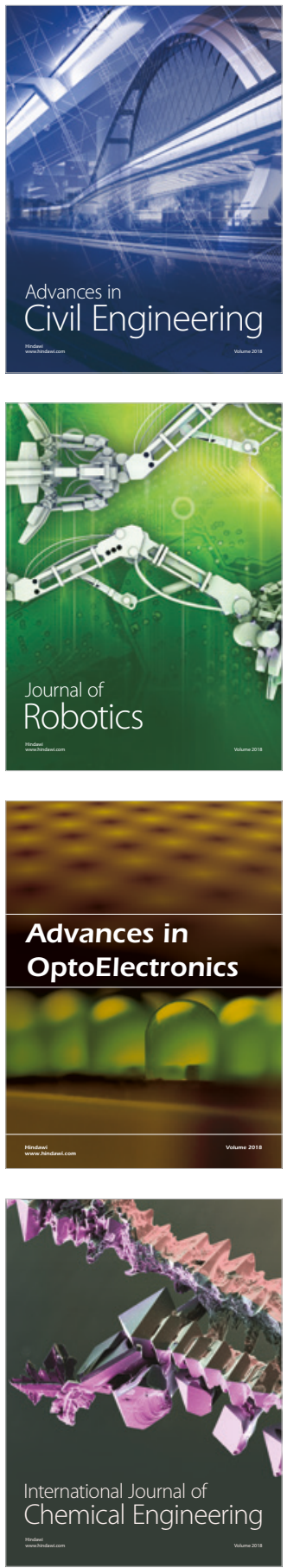

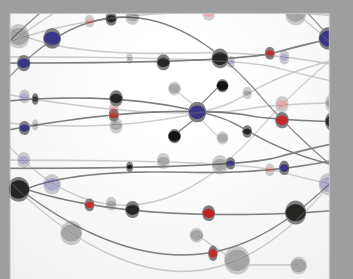

\section{Rotating \\ Machinery}

The Scientific World Journal

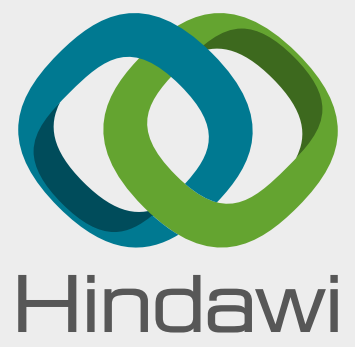

Submit your manuscripts at

www.hindawi.com
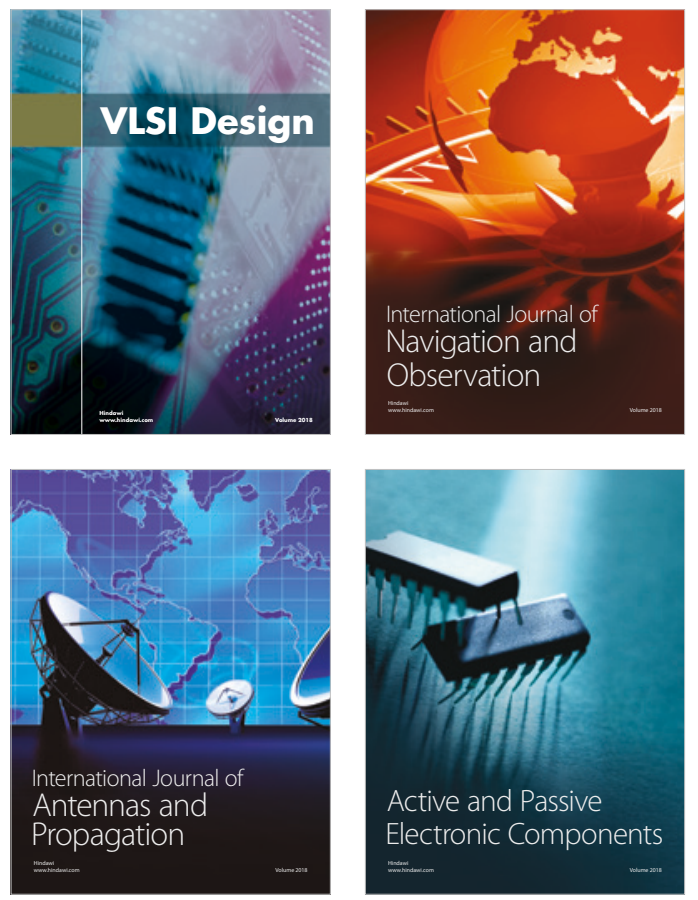
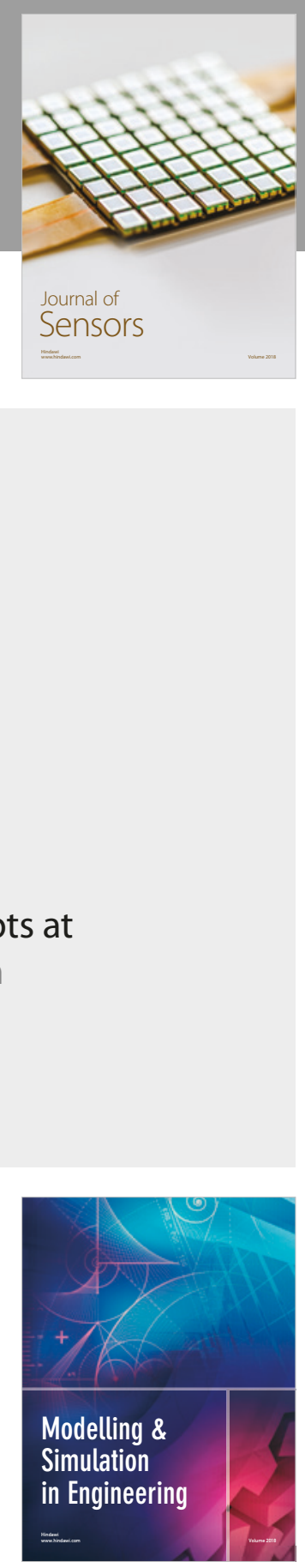

\section{Advances \\ Multimedia}
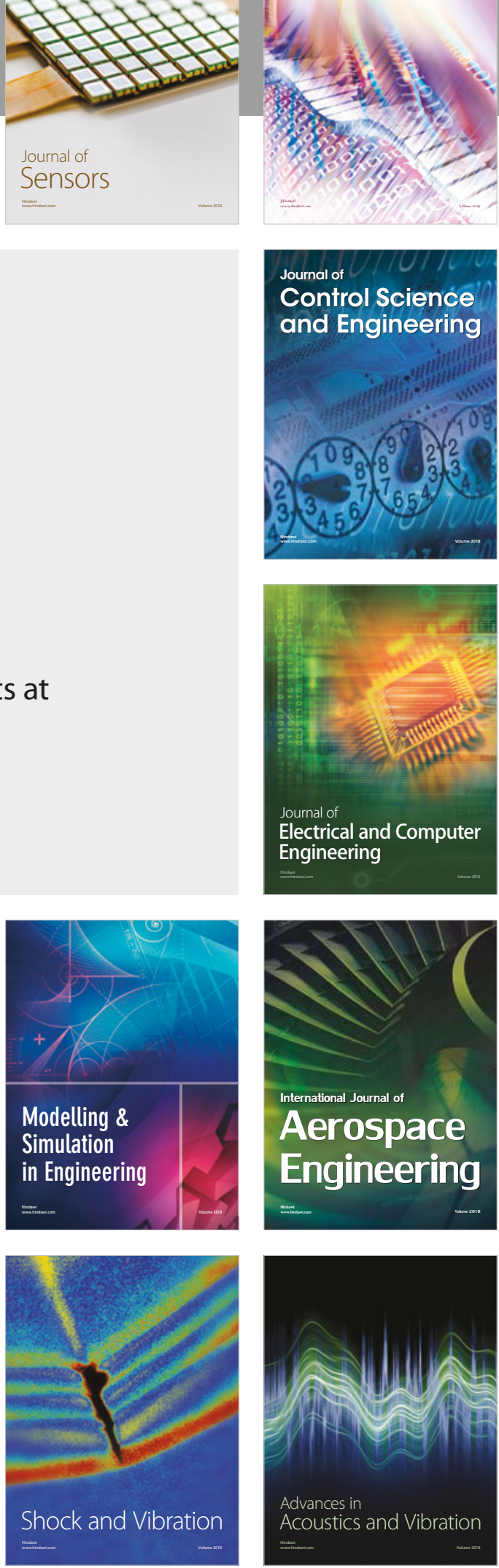